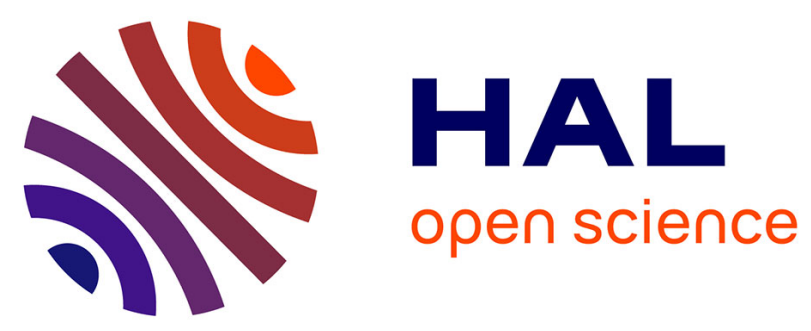

\title{
Actualisation 2018 des recommandations françaises du traitement de l'ostéoporose post-ménopausique
}

Karine Briot, Christian Roux, Thierry Thomas, Hubert Blain, Daniel Buchon, Roland Chapurlat, Françoise Debiais, Jean Marc Feron, Jean Bernard Gauvain, Pascal Guggenbuhl, et al.

\section{To cite this version:}

Karine Briot, Christian Roux, Thierry Thomas, Hubert Blain, Daniel Buchon, et al.. Actualisation 2018 des recommandations françaises du traitement de l'ostéoporose post-ménopausique. Revue du Rhumatisme, 2018, 85 (5), pp.428-440. 10.1016/j.rhum.2018.02.005 . hal-01905211

HAL Id: hal-01905211

https://hal-univ-rennes1.archives-ouvertes.fr/hal-01905211

Submitted on 7 Nov 2018

HAL is a multi-disciplinary open access archive for the deposit and dissemination of scientific research documents, whether they are published or not. The documents may come from teaching and research institutions in France or abroad, or from public or private research centers.
L'archive ouverte pluridisciplinaire HAL, est destinée au dépôt et à la diffusion de documents scientifiques de niveau recherche, publiés ou non, émanant des établissements d'enseignement et de recherche français ou étrangers, des laboratoires publics ou privés. 


\section{Actualisation 2018 des recommandations françaises du traitement de l'ostéoporose post-ménopausique}

Karine Briot 1, Christian Roux 1, Thierry Thomas 2, Hubert Blain ${ }^{3}$, Daniel Buchon ${ }^{4}$, Roland Chapurlat ${ }^{5}$, Françoise Debiais ${ }^{6}$, Jean Marc Feron ${ }^{7}$, Jean Bernard Gauvain 8, Pascal Guggenbuhl 9,10,11, Eric Legrand 12, Anne Marie Lehr-Drylewicz 13, Eric Lespessailles ${ }^{14}$, Françoise Tremollieres ${ }^{15}$, Georges Weryha ${ }^{16}$, Bernard Cortet ${ }^{17}$

${ }^{1}$ Service de rhumatologie, Hôpital Cochin, 75014 Paris, France

2 INSERM U1059, Service de Rhumatologie, CHU de St Etienne, 42100 St Etienne, France.

${ }^{3} \mathrm{CHU}$ de Montpellier, université Montpellier 1, centre Antonin-Balmes, unité de soins aigus gériatriques, 34090 Montpellier, France.

4 Médecine générale, 87032 Limoges, France

5 Service de rhumatologie, Inserm U1033, université de Lyon, hôpital EdouardHerriot, 5, place d'Arsonval, 69437 Lyon, France.

${ }^{6}$ Service de rhumatologie, CHU Poitiers, 86021 Poitiers, France

7 Service de Chirurgie Orthopédique de l'Hôpital Saint Antoine, Hôpitaux Universitaires Est Parisiens, 75012 Paris, France.

${ }^{8}$ Centre de Médecine Gériatrique, 45000 CHR d'Orléans, Orléans, France.

9 Institut NUMECAN, INSERM U 1241, INRA U 1341, 35000 Rennes, France.

${ }^{10}$ Service de Rhumatologie, Hôpital Sud, CHU, 35000 Rennes, France.

${ }^{11}$ Université de Rennes 1, 35000 Rennes, France

12 Service de Rhumatologie, CHU Angers, 49000 Angers, France

${ }^{13}$ Médecine générale, 37000 Tours, France

${ }^{14}$ Service de rhumatologie, CHR Orléans, 45000 Orléans, France

${ }^{15}$ Centre de ménopause, hôpital Paule-de-Viguier, 31300 Toulouse.

${ }^{16}$ Service d'endocrinologie, CHU Nancy, 54000 Nancy, France 
${ }^{15}$ EA 4490, Service de Rhumatologie, CHU Lille, 59000 Lille, France.

Correspondance : Karine Briot,

Hôpital Cochin, Service de rhumatologie, 27, rue du Faubourg Saint-Jacques, 75014 Paris

Email : karine.briot@aphp.fr

Fax : 0158413670 


\section{Résumé}

Objectifs

Actualiser sous l'égide de la section os de la Société française de rhumatologie (SFR) et du groupe de recherche et d'information sur les ostéoporoses (GRIO) en collaboration avec des sociétés savantes (Collège National des Généralistes Enseignants, Collège National des Gynécologues et Obstétriciens Français, Fédération Nationale des Collèges de Gynécologie Médicale, Groupe d'Étude de la Ménopause et du Vieillissement hormonal, Société Française de Chirurgie Orthopédique, Société Française d'Endocrinologie, Société Française de Gériatrie et de Gérontologie) les recommandations du traitement médicamenteux de l'ostéoporose post-ménopausique publiées précédemment en 2012.

\section{Méthodes}

Un groupe de travail, représentatif des spécialités médicales intervenant dans la prise en charge de ces patientes a élaboré ces recommandations à partir d'une analyse systématique de la littérature selon la méthode HAS.

Discussion et conclusion

Ces recommandations insistent sur la prise en charge des femmes avec une fracture sévère chez lesquelles un traitement anti-ostéoporotique est recommandé. En cas de fracture sévère, tous les traitements peuvent être prescrits ; l'acide zolédronique est à privilégier en première intention après une fracture de hanche. Dans les autres cas (avec ou sans fracture non sévère) l'indication thérapeutique dépend des valeurs de la densité minérale osseuse (DMO) et dans les cas difficiles d'outils comme le FRAX®. Tous les traitements peuvent être utilisés ; le raloxifène est à réserver aux patientes à faible risque de fracture périphérique. Le risque de fracture doit réévaluer tous les 2 à 3 ans pour décider des suites de la prise en charge. Ces recommandations abordent le choix du premier traitement et les séquences thérapeutiques

\section{Mots-clés}

Ostéoporose, ménopause, fracture, recommandations, densitométrie osseuse, FRAX 


\section{Objectifs et méthodes}

Ces recommandations sont destinées à tous les médecins amenés à prendre en charge des femmes ayant une ostéoporose post-ménopausique ou à risque d'une telle maladie. Leurs objectifs sont d'exposer les données actuelles d'épidémiologie de la maladie, les éléments clés de l'évaluation des patientes à risque élevé de fracture et les principes du traitement médicamenteux de cette ostéoporose, prenant en compte les données actuelles d'indications, d'efficacité et de tolérance de ces traitements. Le contenu de ces recommandations a été discuté, rédigé en conformité avec la méthode HAS et validé par un groupe de travail pluridisciplinaire. Lorsque les données de la littérature étaient insuffisantes ou incomplètes, les recommandations ont été basées sur un accord professionnel pour prendre en compte l'état des pratiques et les opinions d'experts.

Ces recommandations ne peuvent pas envisager tous les cas spécifiques, toutes les comorbidités, les protocoles de soins hospitaliers, etc. Elles ne revendiquent pas l'exhaustivité des conduites de prise en charge possibles, et ne peuvent se substituer à la responsabilité individuelle du médecin vis-à-vis de son patient. Les indications des médicaments, les remboursements des médicaments, de la densitométrie, du dosage de la $250 \mathrm{H}$ VitD sont valables pour la France.

Ces recommandations ont été écrites par un chargé de projet et un comité scientifique, discutées et relues par un comité de lecture pluridisciplinaire. Plusieurs sociétés savantes : Collège National des Généralistes Enseignants, Collège National des Gynécologues et Obstétriciens Français, Fédération Nationale des Collèges de Gynécologie Médicale, Groupe d'Étude de la Ménopause et du Vieillissement hormonal, Groupe de Recherche et d'Information sur les Ostéoporoses, Société Française de Chirurgie Orthopédique, Société Française d'Endocrinologie, Société Française de Gériatrie et de Gérontologie, et Société Française de Rhumatologie, ont été consultées pour réviser et valider ces recommandations.

\section{2. Épidémiologie de l'ostéoporose et des fractures}

\section{1. Épidémiologie}

L'ostéoporose est une maladie diffuse du squelette caractérisée par la diminution de la résistance osseuse entraînant un risque de fracture (1). L'ostéoporose est un enjeu de santé 
en raison des conséquences graves de certaines fractures dont elle augmente le risque et la fréquence. L'enjeu est d'autant plus important dans les pays dans lesquels l'espérance de vie augmente car l'âge augmente significativement le risque de fracture. Les présentes recommandations s'appliquent quand les causes d'ostéopathies fragilisantes autres que l'ostéoporose post-ménopausique ont été éliminées.

Les fractures ostéoporotiques ou fractures de fragilité surviennent à la suite d'un traumatisme de faible énergie équivalent au plus à une chute de sa propre hauteur en marchant. Tous les os peuvent être le siège d'une fracture ostéoporotique, sauf le crâne, les os de la face, le rachis cervical, les 3 premières vertèbres thoraciques, les mains et les orteils (ces fractures sont alors traumatiques ou tumorales). La chute de sa hauteur est la première cause de traumatisme conduisant à une fracture de fragilité non vertébrale, $5 \%$ des chutes se compliquant de fractures (2), et $2 \%$ d'une fracture de l'extrémité supérieure du fémur (ESF) chez des sujets âgés de plus de 65 ans (3).

\subsubsection{Données épidémiologiques françaises}

En 2001, il a été estimé que chaque année, en France, l'ostéoporose est responsable d'environ 70.000 fractures vertébrales, 60.000 fractures de l'extrémité supérieure du fémur (ESF) et 35000 fractures du poignet (4). Les données récentes montrent que le nombre de patients opérés pour fracture de l'ESF augmente depuis 2002 de $+5 \%$ chez les femmes (de 49285 en 2002 à 50215 en 2013) et +22\% chez les hommes, (de 12716 en 2002 à 15482 en 2013) (5). Le rapport de la DREES (Direction de la Recherche, des Etudes, de l'Evaluation et des Statistiques) publié en janvier 2016 montre qu'en France, toutes causes confondues, 23,5\% des patients de 55 ans ou plus meurent dans l'année qui suit une fracture de l'ESF (www.data.drees.sante.gouv.fr).

La Caisse Nationale d'Assurance Maladie (France), a analysé les hospitalisations pour fracture après 50 ans, pour le territoire français, au cours de l'année 2013 (6). En 2013, 177000 patients de plus de 50 ans ont été hospitalisés pour une fracture ostéoporotique (tous sites anatomiques confondus). Ces hospitalisations concernaient 3 femmes pour 1 homme; les patients sont âgés de plus de 70 ans pour 2/3 d'entre eux. Le nombre d'hospitalisations pour fracture a augmenté de $9 \%$ entre 2011 et 2013 , quel que soit le type de fracture. Le coût direct est de 771 millions d'euros par an. Douze mois plus tard, 6325 patients sont décédés (7\%), $12 \%$ ont présenté une nouvelle fracture, $40 \%$ ont été hospitalisés une deuxième fois. Le taux de décès est deux fois plus important chez les hommes que chez les femmes. Douze mois plus tard, seulement $10 \%$ des patients ont eu une ostéodensitométrie et $15 \%$ un traitement de fond de l'ostéoporose. Plus de $80 \%$ des 
patients avec fractures ne sont donc pas pris en charge dans les suites de la fracture en France (6), et ceci est comparable aux données internationales (7).

\subsubsection{Conséquences des fractures sévères}

Certaines fractures dites sévères sont associées à un excès de mortalité ; ce sont les fractures de l'extrémité supérieure du fémur (FESF), de l'extrémité supérieure de l'humérus (FESH), des vertèbres, du pelvis, du bassin ou sacrum, de la diaphyse fémorale et du fémur distal, des 3 côtes simultanées et du tibia proximal (8-10). L'excès de mortalité par rapport à la population générale est surtout observé chez les sujets âgés de moins de 70 ans (8). Des études épidémiologiques ont confirmé l'excès de mortalité après fractures du bassin et après fractures de l'humérus (11). Les fractures sévères sont également associées à un risque de refractures vertébrales et non vertébrales, cette nouvelle fracture expliquant $25 \%$ de l'excès de mortalité (12).

D'autres fractures fréquentes, dites non sévères, comme celles des os de l'avantbras ont un retentissement important mais ne sont pas associées à un excès de mortalité. Elles peuvent cependant être révélatrices d'une ostéoporose et sont associées au risque de refracture, en particulier sévère (13).

\subsection{Facteurs de risque de fracture et risque de fracture à court terme}

Le caractère récent d'une fracture est un facteur de risque majeur de refracture à court terme. C'est notamment le cas des sujets avec fractures vertébrales dont le risque de nouvelle fracture est de $25 \%$ dans l'année qui suit (14). Le risque de fracture est également majoré dans les 2 à 3 ans qui suivent une fracture non vertébrale $(15,16)$. Les facteurs de risque de refracture à court terme sont, en plus, de la survenue d'une fracture récente, la présence de facteurs de risque de chute $(17,18)$. Les patients ayant ces facteurs de risque nécessitent une prise en charge rapide pour diminuer le risque d'une $2^{\mathrm{e}}$ fracture.

\subsection{Comorbidités et risque de fracture}

Les patients ayant des fractures ostéoporotiques sont plus souvent les patients ayant des maladies chroniques, par exemple les maladies neurologiques (pathologies démentielles et maladie de Parkinson en particulier), le diabète, les maladies cardio-vasculaires, etc. et polymédicamentés $(19,20)$. II est nécessaire de prendre en compte ces comorbidités car elles augmentent le risque de fracture et les conséquences des fractures(en particulier la 
fracture du col du fémur chez un sujet dément, ou la fracture vertébrale chez un sujet ayant une bronchite post-tabagique chronique obstructive). II est important d'évaluer le bénéfice/risque chez ces patients et de décider avec le patient d'un traitement et du mode d'administration.

\section{3. Évaluation du risque de fracture et décision thérapeutique}

La décision de proposer un traitement anti-ostéoporotique est prise en fonction de l'antécédent personnel de fracture, de son type, de son ancienneté, de l'âge, des antécédents du patient en particulier les facteurs de risque de chute et du résultat de la mesure de la densité minérale osseuse (DMO).

\subsection{Outils de prédiction du risque de fracture}

\subsubsection{Antécédent personnel de fracture}

C'est le facteur de risque le plus important de nouvelle fracture $(14,15,18,21,22)$. Ceci est vrai pour tous les types de fractures, y compris non vertébrales. Le risque de fracture est augmenté surtout dans les 2 à 3 ans qui suivent la fracture, et reste significatif jusqu'à 10 à 15 ans après la survenue de la première fracture, (en particulier vertèbres et humérus) $(22,23)$. II convient de distinguer fracture récente et plus ancienne, la fracture récente étant associée à un risque plus élevé de fracture à court terme.

La fracture vertébrale est fréquente mais sous-estimée (dans 2/3 des cas) en raison de son caractère parfois peu symptomatique ou de douleurs banalisées, prises pour une pathologie disco-vertébrale commune et ne conduisant pas à la réalisation d'une radiographie. C'est pourtant un facteur de risque essentiel d'autres fractures, vertébrales et périphériques. Le risque de nouvelle fracture augmente avec le nombre et la sévérité des fractures vertébrales. La découverte d'une fracture vertébrale radiographique, même asymptomatique au moment de la découverte, augmente le risque relatif de fractures incidentes dès la première année et jusqu'à 15 ans après la découverte de la fracture après ajustement pour l'âge et la densité osseuse (21).

Une évaluation morphologique à la recherche de fractures vertébrales par radiographies standards du rachis dorso-lombaire ou VFA (Vertebral Fracture Assessment) (non inscrit à la 
nomenclature des actes médicaux) réalisée par les appareils d'absorptiométrie biphotonique à rayons- $X$, est indiquée chez la femme ménopausée en cas de rachialgies ou si un des critères suivants est présent : perte de taille $\geq 4 \mathrm{~cm}$ (mesure de la taille comparée à la taille rapportée à l'âge de 20 ans), perte de taille prospective $\geq 2 \mathrm{~cm}$ (mesure répétée de la taille au cours du suivi), antécédent de fracture vertébrale et maladies chroniques et traitements (corticothérapie, inhibiteurs de l'aromatase) avec risque important de fracture vertébrale (ISCD 2015) (www.iscd.org) ( Accord professionnel).

\subsubsection{La mesure de la densité minérale osseuse :}

L'ostéodensitométrie par absorptiométrie biphotonique à rayons-X (DXA) est la technique de référence pour mesurer la densité minérale osseuse (DMO) au rachis lombaire et à l'extrémité supérieure du fémur. La résistance osseuse est fortement dépendante de la $\mathrm{DMO}$. Chez les femmes ménopausées, le résultat s'interprète en T-score, différence entre la densité osseuse mesurée et la densité osseuse moyenne des femmes jeunes au même site osseux; il s'exprime en unités d'écart-type. Il a été proposé par l'OMS une définition densitométrique de l'ostéoporose : $T \leq-2,5$ au col fémoral (OMS) (24). Cet examen est

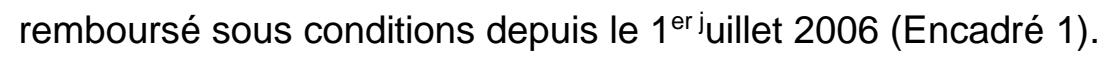

\subsubsection{Choix du site et de la courbe}

Il est préconisé de mesurer la DMO à 2 sites : le rachis lombaire et l'extrémité supérieure du fémur (col fémoral, hanche totale). II n'y a aucune indication à mesurer le radius dans l'ostéoporose post-ménopausique (25). Selon I'IOF (International Osteoporosis Foundation), si un seul site doit être privilégié, ce doit être la hanche totale ou le col fémoral en utilisant 1 seule courbe de référence NHANES III $(26,27)$. La mesure de DMO au col du fémur est la plus fortement associée au risque de fracture dans l'ensemble des cohortes, est intégrée dans le FRAX et peut être le site choisi pour la cible thérapeutique lors du suivi des traitements.

\subsubsection{Relation entre T-score bas et risque de fracture}

Il existe un gradient de risque entre une valeur abaissée de DMO et une augmentation du risque de fracture. Pour toute diminution d'une déviation standard de la DMO, le risque de fracture est multiplié par deux (28-30). Plus la valeur de T-score diminue, plus le risque de fracture ostéoporotique et de hanche augmente $(31,32)$. La diminution de la DMO à tous les sites est associée au risque de tous types de fracture; cependant la 
diminution de la DMO mesurée au fémur est fortement prédictive du risque de tout type de fracture, et de fracture du fémur $(32,33)$.

\subsubsection{Limites de l'utilisation d'une valeur seuil de T-score}

Une définition densitométrique de l'ostéoporose ne permet pas d'identifier toutes les femmes à risque de fracture ; plus de $50 \%$ des fractures périphériques surviennent chez des patients ayant un T-score $>-2.5$ (30, 34, 35,36). Parmi ces patients avec une ostéoporose non densitométrique objectivée par la survenue de ces fractures non traumatiques on peut identifier des altérations spécifiques du tissu osseux responsables de sa fragilité excessive : densité osseuse inadaptée au poids chez les sujets obèses ; matrice osseuse anormale en raison de la glycation des protéines chez les diabétiques; excès de résorption osseuse, chez les femmes débutant un traitement par inhibiteurs de l'aromatase (37-41), etc.

\subsection{3. Évaluation du risque de chutes}

Les facteurs de risque de chutes sont des facteurs déterminants dans la survenue de fractures non vertébrales chez les sujets les plus âgés et/ou fragiles (42). Des recommandations sur les modalités de dépistage des sujets à risque de chute ont été $\begin{array}{lllll}\text { élaborées } & \text { par } & \text { l'HAS } & \text { en } & \end{array}$ sante.fr/portail/upload/docs/application/pdf/prevention_des_chutes-argumentaire.pdf).

Un grand nombre de facteurs prédisposent au risque de chute (Tableau $\left.{ }^{\circ} 1\right)$. Parce qu'il est difficile d'envisager une analyse et une prise en charge des facteurs de risque de chute chez tous les sujets âgés, il est recommandé, par la HAS, mais aussi dans les recommandations anglo-américaines de 2010 et par d'autres sociétés savantes, de faire un repérage des personnes âgées à risque par des tests et questions simples. En pratique, un antécédent de chute quelles qu'en soient les conditions et d'autant plus qu'il est récent (dans les 3 à 6 derniers mois en particulier) ou une peur de tomber réduisant l'autonomie fonctionnelle doivent inciter à proposer une évaluation des causes de déséquilibre, éventuellement via une consultation gériatrique spécialisée. En cas de doute, la présence de troubles de l'équilibre dynamique, authentifiée par un Timed up and go test supérieur à 14 secondes ou d'équilibre statique (temps d'appui unipodal supérieur à 5 secondes, difficulté à garder l'équilibre en cas de poussée sternale par exemple) confirmera la nécessité d'un bilan étiologique et d'une prise en charge adaptée $(43,44)$.

\subsection{Les outils d'évaluation du risque de fracture pour le spécialiste}


Ces outils peuvent être utiles à tous les médecins qui connaissent la prise en charge de la pathologie osseuse et l'interprétation des résultats de ces outils.

3.2.1. L'estimation du risque absolu de fracture par le FRAX $®$ :

L'identification des sujets à risque de fracture repose sur une évaluation multifactorielle qui comprend la mesure de la DMO et l'évaluation de facteurs de risque cliniques associés au risque de fracture (Encadré²). Le rôle de ces différents facteurs de risque est différent suivant l'âge. L'outil $F R A X{ }^{\circledR}$ a été proposé pour la quantification du risque de fracture (45) (www.sheffield.ac.uk/FRAX). Le résultat est une probabilité à dix ans de fracture de l'extrémité supérieure du fémur et des fractures dites « majeures » regroupant celles de l'extrémité supérieure du fémur, de l'humérus, du poignet ainsi que les fractures vertébrales cliniques. L'outil FRAX a été testé dans différentes cohortes françaises (46-50). Compte tenu des études de validation ou de calibration nationales (46-50) et des recommandations internationales (NOF, NOS, NOGG) les recommandations suivantes sont proposées (Accord professionnel) :

- Le calcul du FRAX® n'est pas utile chez les sujets pour lesquels l'indication de traiter est évidente comme l'antécédent de fracture sévère et/ou $T \leq-3$ au site vertébral et fémoral (fémur total ou col fémoral).

- Le seuil d'intervention choisi est fonction de l'âge. Pour un âge donné, la valeur seuil de FRAX $®$ à partir de laquelle il est proposé de débuter un traitement anti-ostéoporotique correspond au risque calculé des femmes de même âge ayant déjà fait une fracture (risque de récidive) (45). Cette valeur seuil en fonction de l'âge est représentée sur la figure 1 (Accord professionnel).

\subsubsection{Le TBS}

Le Trabecular bone score (TBS) est un paramètre de texture osseuse mesuré automatiquement sur les données d'ostéodensitométrie au rachis lombaire. Le TBS est plus bas chez les patients qui ont des fractures de fragilité : plusieurs études ont montré sa capacité discriminante chez de tels patients. La prédiction du risque de fracture par la combinaison de la DMO et du TBS est meilleure que la mesure de DMO seule au rachis lombaire. Cependant dans les études prospectives, le meilleur facteur prédictif reste la mesure de DMO au site fémoral. La mesure systématique du TBS n'est pas recommandée dans la prédiction du risque de fracture et le suivi des traitements car sa capacité à reclasser les patients n'est pas suffisamment établie $(51,52)$. Le TBS est également associé au risque 
de fractures ostéoporotiques et de hanche après ajustement sur le $\operatorname{FRAX}(53,54)$. Une méta-analyse de 14 études prospectives a montré que l'ajustement du FRAX® sur le TBS améliorait un peu la valeur prédictive du $\operatorname{FRAX®~}(55,56)$. En dehors de situations où la décision thérapeutique est simple, le FRAX TBS peut être utilisé comme peut l'être le FRAX avec le même seuil de décision thérapeutique que pour le FRAX.

\subsubsection{Les marqueurs du remodelage osseux}

Le remodelage osseux peut être évalué de manière non invasive par différents paramètres biologiques. Certains sont spécifiques de la formation (ostéocalcine, phosphatase alcaline osseuse, peptides d'extension du procollagène I), et d'autres de la résorption osseuse (peptides de dégradation). Les limites de ces marqueurs portent sur leur interprétation clinique qui doit prendre en compte des facteurs de confusion comme la fonction rénale ou l'existence d'une fracture récente. À I'heure actuelle, il n'y a pas de marqueurs qui pourraient prédire le résultat de l'ostéodensitométrie. Ils peuvent en revanche prédire la perte osseuse. La combinaison de plusieurs marqueurs entre eux et/ou avec d'autres facteurs de risque pourrait améliorer leur pertinence dans la prédiction de la fragilité osseuse. Les études publiées ne retrouvent pas de façon reproductible de marqueur qui soit associé avec une augmentation du risque de fracture bien que l'utilisation conjointe des marqueurs avec la mesure de la DMO puisse améliorer la prédiction. Des recommandations récentes d'experts ont jugé insuffisantes les preuves de l'intérêt des marqueurs pour la prédiction du risque de fracture en pratique clinique (57). Leur utilisation n'est donc pas recommandée en routine dans cette indication mais son utilisation peut aider les spécialistes à décider d'un traitement dans des situations difficiles. Par ailleurs, ils sont utiles pour le suivi des traitements inhibiteurs de la résorption osseuse.

\section{Stratégies thérapeutiques pour la prévention et le traitement}

\section{de l'ostéoporose post-ménopausique}

Le diagnostic d'ostéoporose nécessite d'éliminer les autres causes d'ostéopathies fragilisantes (métabolique, maligne et génotypique). Cette démarche diagnostique est nécessaire avant tout traitement. L'objectif du traitement étant de prévenir la survenue de fractures, la diminution du risque de fracture passe par le renforcement de la solidité (ou résistance) du tissu osseux et la prévention des chutes. La prise en charge est donc à la fois 
pharmacologique et non pharmacologique. Les preuves du bénéfice anti-fracturaire des médicaments de l'ostéoporose ont été établies dans des populations définies comme ostéoporotiques par des critères densitométriques ou par l'existence d'une fracture (Tableaux 2 et 3) (58-75).

II n'y a pas de données permettant de comparer directement l'efficacité anti-fracturaire des traitements. Les données densitométriques et biochimiques ne peuvent pas être utilisées pour cette comparaison. Le choix du traitement doit tenir compte des effets extra-osseux bénéfiques ou indésirables, des contre-indications spécifiques des médicaments, des contraintes des traitements et de la décision partagée avec le patient. Le respect des conditions de prise des médicaments peut contribuer à limiter la survenue de certains effets indésirables. Le traitement doit être choisi en fonction de l'âge, du risque de fracture vertébrale et/ou périphérique, de la sévérité de la fracture (Accord professionnel), dans le respect des conditions de remboursement.

Dans tous les cas, la patiente sera informée sur sa maladie et les traitements. L'importance d'une bonne adhésion au traitement sera expliquée dans une démarche de décision partagée avec la patiente, et cet élément sera surveillé tout au long du suivi.

En cas de difficulté ou d'échec d'un traitement médicamenteux, l'avis d'un spécialiste de pathologies osseuses doit être pris (Accord professionnel). La prise en charge en réseau multidisciplinaire (filière fracture) a montré son intérêt dans une meilleure prise en charge des malades fracturés $(76,77)$. Dans ces recommandations seront envisagés à la fois la première séquence thérapeutique et les relais de traitements. L'encadré 3 présente les exemples de recommandations thérapeutiques dans différentes situations cliniques.

\subsection{Traitement médicamenteux de l'ostéoporose}

\subsubsection{Recommandations en cas de fracture}

\subsubsection{En cas de fracture sévère}

La réalisation d'une ostéodensitométrie est recommandée avant toute décision thérapeutique si la situation médicale le permet (Grade A).

Un traitement est recommandé quel que soit l'âge après une fracture de fragilité sévère (extrémité supérieure du fémur, vertèbre, fémur distal, extrémité supérieure de l'humérus, bassin, tibia proximal) si le T score est inférieur ou égal à -1 (Accord professionnel) (Fig. 2) 
Dans ces situations, la mesure d'ostéodensitométrie ou l'ostéodensitométrie permet de quantifier la fragilité osseuse sous-jacente, vérifier que le T-score est inférieur à -1, et planifier le suivi thérapeutique (Fig. 2). En cas de T-score $>-1$, l'avis d'un spécialiste de pathologie osseuse et l'usage d'outils de prédiction (FRAX, TBS, marqueurs du remodelage osseux), peut-être recommandé.

En cas de fracture sévère hors fracture vertébrale les traitements remboursés sont :

- acide alendronique (alendronate) $70 \mathrm{mg}$ hebdomadaire (ou $10 \mathrm{mg} / \mathrm{j}$ ),

- acide risédronique (risédronate) 35 mg hebdomadaire ou 75 mg 1 comprimé 2 jours de suite 1 fois par mois (ou $5 \mathrm{mg} / \mathrm{j}$ ),

- acide zolédronique (zolédronate) $5 \mathrm{mg} 1$ perfusion une fois par an. C'est le seul traitement ayant démontré son efficacité chez les patientes ayant eu une fracture de l'ESF (69)

- denosumab $60 \mathrm{mg} 1$ injection SC tous les 6 mois, remboursé en relais des bisphosphonates.

En cas de fractures vertébrales : les traitements remboursés sont :

- acide alendronique (alendronate) $70 \mathrm{mg}$ hebdomadaire (ou $10 \mathrm{mg} / \mathrm{j}$ ),

- acide risédronique (risédronate) 35 mg hebdomadaire ou 75 mg 1 comprimé 2 jours de suite 1 fois par mois (ou $5 \mathrm{mg} / \mathrm{j}$ ),

- acide zolédronique (zolédronate) $5 \mathrm{mg} 1$ perfusion une fois par an,

-denosumab $60 \mathrm{mg} 1$ injection SC tous les 6 mois, remboursé en relais des bisphosphonates,

- raloxifène $(60 \mathrm{mg} / \mathrm{j})$, remboursé jusqu'à 70 ans

- tériparatide $20 \mathrm{ug} / \mathrm{j}$ remboursé avec au moins 2 fractures vertébrales,

- Traitement Hormonal de la Ménopause (THM) entre 50 et 60 ans si troubles du climatère.

L'utilisation des médicaments à voie d'administration parentérale (acide zolédronique, denosumab peut être privilégiée dans les situations suivantes: fractures de l'extrémité supérieure du fémur (FESF), DMO très basse, présence de comorbidités et notamment troubles mnésiques, défaut d'observance et polymédication : (Accord professionnel).

\subsubsection{2. $\quad$ En cas de fractures non sévères (poignet et autres sites)}

La réalisation d'une ostéodensitométrie est recommandée avant toute décision thérapeutique (Grade A). Compte tenu du gradient de risque entre une valeur abaissée de 
DMO et une augmentation du risque de fracture, les indications thérapeutiques dépendent du T score et sont décrites dans le tableau 7 (29-31) (Accord professionnel).

Un traitement est recommandé si le T-score est inférieur ou égal à -2 à au moins un des sites (rachis, fémur). En cas de T-score $>-2$ et $<-1$, l'avis d'un spécialiste de pathologie osseuse et l'usage d'outils de prédiction (FRAX, TBS, marqueurs du remodelage osseux), peut être recommandé. En cas de T-score >-1; le traitement n'est pas recommandé (Accord professionnel).

Lorsqu'un traitement anti-ostéoporotique est indiqué, les possibilités thérapeutiques sont (par ordre alphabétique) :

- acide alendronique (alendronate) $70 \mathrm{mg}$ hebdomadaire (ou $10 \mathrm{mg} / \mathrm{j}$ ),

- acide risédronique (risédronate) 35 mg hebdomadaire ou 75 mg 1 comprimé 2 jours de suite 1 fois par mois (ou $5 \mathrm{mg} / \mathrm{j}$ ),

- acide zolédronique (zolédronate) $5 \mathrm{mg} 1$ perfusion une fois par an,

- denosumab $60 \mathrm{mg} 1$ injection SC tous les 6 mois, remboursé en relais des bisphosphonates

- raloxifène $(60 \mathrm{mg} / \mathrm{j})$,

- Traitement Hormonal de la Ménopause (THM) entre 50 et 60 ans en présence de troubles du climatère.

L'usage du raloxifène : il est à réserver aux patientes avec un risque de fracture périphérique peu élevé (Grade A) : âge inférieur à 70 ans ou absence des facteurs de risque suivants : T score fémoral $\leq-3$, risque de chute élevé, antécédent de fracture périphérique).

L'usage du THM : il est indiqué s'il existe des troubles climatériques chez les femmes ménopausées âgées de moins de 60 ans, le traitement ayant fait la preuve la preuve de son efficacité en début de ménopause. La durée de sa prescription est fonction de ces troubles, après discussion du rapport bénéfice/risque avec la patiente. En l'absence de troubles climatériques, le THM peut être prescrit en cas d'intolérance ou inefficacité des autres traitements à visée osseuse. Si les doses utilisées sont inférieures aux doses recommandées pour la protection osseuse, une ostéodensitométrie doit être répétée après 2 ou 3 ans de traitement (Grade A). Si la DMO reste basse, il est possible d'ajouter un traitement anti-ostéoporotique au THM.

\subsubsection{Recommandations en l'absence de fracture}

Il est recommandé de rechercher une ostéoporose par ostéodensitométrie chez les femmes ménopausées ayant des facteurs de risque d'ostéoporose (Grade A). Dans ce cas, cette ostéodensitométrie est remboursée. Parce que les chutes et l'ostéoporose sont deux 
facteurs de risque de fracture non vertébrale indépendants et qu'une ostéoporose est fréquemment trouvée chez les chuteurs, il est recommandé de rechercher une ostéoporose par ostéodensitométrie chez les sujets âgés à risque de chute (Accord professionnel) (78).

Compte tenu du gradient de risque entre une valeur abaissée de DMO et une augmentation du risque de fracture, Les indications thérapeutiques, dépendent du T score et sont décrites dans le tableau 7 (29-31) (Accord professionnel).

Un traitement est recommandé si le T-score est inférieur ou égal à -3 à au moins un des sites (rachis, fémur). En cas de T-score $>-3$ et $\leq-2$, l'avis d'un spécialiste de pathologie osseuse et l'usage d'outils de prédiction (FRAX, TBS, marqueurs du remodelage osseux), peut être recommandé. En cas de T-score >-2 ; le traitement n'est pas recommandé.

Lorsqu'un traitement est indiqué, les possibilités thérapeutiques sont celles décrites en cas de fractures non sévères.

Les conditions d'usage du raloxifène et du THM ont été décrites dans le paragraphe précédent.

\subsection{Mesures thérapeutiques associées au traitement de l'ostéoporose}

\subsubsection{Prévention des chutes}

Chez les sujets âgés ou fragiles, il faut souligner l'importance de la prévention des chutes et de leurs conséquences. En effet, la mise en place de mesures adaptées chez les patients âgés à risque de chute permet de réduire le risque de chute chez les patients âgés vivant à leur domicile (79). On peut citer tout particulièrement: l'activité physique permettant de renforcer l'équilibre, la supplémentation en vitamine $D$ en cas de taux sérique insuffisant, la réduction des médicaments responsable de troubles de vigilance ou d'hypotension orthostatique, la réduction des dangers environnementaux, l'amélioration de la vision et la prise en charge adaptée des douleurs des membres inférieurs

L'évaluation individuelle du risque de chute et la prise en charge adéquate des patients nécessitent la collaboration de réseaux de soins (filières fractures et orthogériatriques) avec les services de rééducation et les équipes de gériatrie.

L'inclusion dans les programmes d'activité physique comprenant un entrainement spécifique visant à améliorer l'équilibre, est un facteur clé de succès vis-à-vis du risque de chute. II doit être bien entendu associé à d'autres types d'exercices (renforcement musculaire, travail de la coordination et de l'endurance, augmentation des amplitudes articulaires, en particulier de cheville) (79) qui ont montré leur efficacité pour diminuer le risque de chute ou le risque de chute compliquée (Grade A). 
Chez les plus de 65 ans il est recommandé de pratiquer une activité physique d'intensité modérée à élevée, 2 jours par semaine ou plus, de préférence non consécutifs, à raison de 8 à 10 exercices répétés 8 à 12 fois chacun (Actualisation des repères du PNNS (Programme National Nutrition Santé) - Révisions des repères relatifs à l'activité physique et à la sédentarité 2016)) (www.anses.fr).

Les freins à la pratique de l'activité physique restent multiples. Alors que l'âge et les problèmes de santé sont souvent les raisons invoquées pour ne pas pratiquer d'activité physique, il est important de souligner au contraire que ces raisons sont justement celles qui justifient la nécessité d'inciter et d'accompagner vers la pratique d'une activité physique. Une durée d'exercice n'est pas recommandée mais les exercices doivent être réalisés jusqu'au moment où il devient difficile de les répéter encore une fois, sans aide. Ainsi, la qualité et l'intensité de l'activité physique ont toutes deux leur importance, mais au final il faut également insister auprès des sujets âgés et de leurs aidants sur la complémentarité et le cumul des effets bénéfiques de la réduction de la sédentarité et du programme d'activité physique pour diminuer le risque de chute sévère et dans certaines études le risque de fracture (Grade B) (80-82).

\subsubsection{Apports calciques}

Les apports quotidiens recommandés doivent être d'au moins $1 \mathrm{~g}$ à $1,2 \mathrm{~g}$ chez les femmes ménopausées âgées de plus de 50 ans, en privilégiant les apports alimentaires (produits laitiers et eaux minérales riches en calcium) (Accord professionnel). Dans un contexte de carence, chez les patients à risque de fracture, les apports quotidiens recommandés doivent être d'au moins 1 gramme selon le PNSS. En pratique, il est possible d'évaluer les apports alimentaires par un auto-questionnaire fréquentiel disponible en ligne (www.grio.org) [Annexe A, Table S1 ; voir le matériel complémentaire accompagnant la version en ligne de cet article]. L'efficacité de la supplémentation calcique administrée seule, dans le but de prévenir les fractures ostéoporotiques n'est pas démontrée. L'association d'une incidence accrue des événements cardiovasculaires et de la supplémentation calcique chez les femmes âgées a été suggérée (83-85). Elle a été rapportée essentiellement chez les sujets dont l'apport calcique alimentaire spontané était déjà suffisant (86). En cas d'insuffisance, une supplémentation en vitamine D doit être associée.

\subsubsection{La Vitamine D}

La concentration recommandée actuellement de $25 \mathrm{OH}$ vitamine $\mathrm{D}$ est d'au moins $30 \mathrm{ng} / \mathrm{ml}$ (75 nmol/l) (Accord professionnel) (45). Un dosage de vitamine $D$ doit être réalisé afin d'éliminer une autre cause d'ostéopathie fragilisante (ostéomalacie) et chez les sujets chuteurs devant recevoir un traitement anti-ostéoporotique. Ces deux situations rentrent dans le cadre du remboursement de ce dosage. L'existence d'un taux cible nécessite parfois de vérifier le dosage sérique de vitamine $D$ au cours du suivi en particulier dans les 
populations à risque (morbidité, malabsorption, difficulté à atteindre l'objectif thérapeutique, insuffisance profonde $<10 \mathrm{ng} / \mathrm{ml}$ en vitamine D...). Cette recommandation s'applique aux patients nécessitant un traitement anti-ostéoporotique (Accord professionnel).

En cas d'insuffisance ou de carence vitaminique D, un traitement "d'attaque » peut permettre d'obtenir rapidement un taux de $25-(\mathrm{OH})$-vitamine $\mathrm{D}$ au-dessus de la valeur cible de $30 \mathrm{ng} / \mathrm{ml}(45)$.

La posologie du traitement d'entretien est de 800 à $1200 \mathrm{UI} / \mathrm{jour}$. Des doses équivalentes de 80000 à $100000 \mathrm{UI}$ tous les 2 à 3 mois peuvent être proposées (45). Les posologies fortes (500 000 ou 600000 UI, une à deux fois par an), ne sont pas recommandées dans l'état actuel des connaissances, car elles pourraient être délétères (87) (Accord professionnel).

\subsection{Eviction des facteurs de risque modifiables}

À chaque fois qu'elle est possible l'éviction des facteurs de risque de fractures et de chutes est nécessaire: sevrage du tabac et des médicaments non indispensables (opiacés, hypnotiques) $(88,89)$, arrêt ou dose minimale efficace des glucocorticoïdes par voie orale, diminution de la consommation d'alcool, etc.

\section{Suivi de l'ostéoporose}

Les principes du suivi en fonction du traitement anti-ostéoporotique sont décrits dans le tableau 4.

\subsection{Suivi Clinique}

Le suivi clinique est indispensable (Accord professionnel). II comporte le recueil des événements fracturaires, la recherche de nouveaux facteurs de risque et/ou maladies inductrices d'ostéoporose, l'évaluation du risque de chutes (chutes récentes, facteurs de risque de chutes), la tolérance et l'observance des traitements.

La mesure de la taille: les fractures vertébrales sont responsables d'une diminution de la taille. La réduction de la taille (à mesurer une fois par an chez les sujets ostéoporotiques) est un signe d'alerte non spécifique de pathologie rachidienne (90-92).

Les traitements de l'ostéoporose, comme ceux de toute maladie chronique, ne sont efficaces qu'en cas d'adhésion optimale et plusieurs études ont montré la moindre efficacité 
des traitements en cas de mauvaise adhésion (93). Le suivi de l'adhésion aux traitements ainsi qu'aux recommandations de prévention des chutes pour les plus âgées est donc un élément indispensable dans le suivi clinique.

Les patientes traitées pour ostéoporose doivent être informées du risque très faible d'ostéonécrose de la mâchoire et de fracture atypique fémorale sous traitement par bisphosphonates et denosumab. II est recommandé d'effectuer les éventuels soins dentaires nécessaires au début du traitement. Si le patient bénéficie d'un suivi régulier par un dentiste et en l'absence d'avulsion dentaire ou autre geste dentaire profond prévue à court terme, un traitement par antirésorbeur peut être débuté. Si le patient n'a pas de suivi dentaire il est recommandé de consulter un dentiste. En cas de risque élevé de fracture à court terme comme après une fracture sévère, l'évaluation dentaire ne doit pas retarder la mise en route d'un traitement. Le suivi bucco-dentaire sous traitement est identique à celui recommandé dans la population générale, au minimum une fois par an. II est possible d'effectuer des avulsions dentaires, lorsqu'elles sont nécessaires, sous traitement antibiotique. La prise de bisphosphonate ou denosumab pour une ostéoporose n'est pas une contre-indication à la mise en place d'un implant dentaire (http: //afssaps.sante.fr/htm/10/filltrpsc/lp071203.htm) (www.sscmfco.fr). Ces recommandations ne s'appliquent pas aux sujets traités par bisphosphonates ou denosumab dans le cadre d'une ostéolyse tumorale.

\subsection{Place de la mesure de la DMO au cours du suivi}

\subsubsection{Rythme des examens}

Une mesure de la DMO peut être réalisée dans les 2 à 3 ans après le début du traitement et à chaque fois qu'est envisagée une modification du traitement (arrêt ou changement) : le but est de vérifier l'absence de perte osseuse (définie par une diminution de plus de $0,03 \mathrm{~g} / \mathrm{cm}^{2}$ de DMO) (94) (Grade B). Un arrêt précoce d'un traitement pour effet indésirable justifie également la réalisation d'une nouvelle mesure de DMO.

Les données récentes montrent qu'avec l'acide zolédronique, la part attribuable au gain en DMO à la hanche totale dans la réduction du risque de fracture vertébrale et non vertébrale est de 40 à $61 \%$ (95). Avec le dénosumab l'effet anti-fracturaire à 3 ans est expliqué à plus de $50 \%$ pour les fractures vertébrales, et à $72 \%$ pour les fractures non vertébrales, par l'augmentation de la densité osseuse aux mêmes sites (96). II en va de même pour le tériparatide. Ces données montrent par conséquent que la répétition de la densité osseuse 
n'a plus seulement pour but de dépister des non répondeurs aux traitements, mais d'évaluer la réponse osseuse aux traitements, dans une perspective de suivi thérapeutique strict.

Le résultat de la DMO en fin de séquence est un des éléments qui permet d'évaluer le risque de fracture dans les années qui suivent. En effet la mesure de DMO fémorale après 5 ans de traitement par alendronate ou 3 ans de traitement par acide zolédronique, permet d'évaluer le risque de fracture pour les années suivantes $(97,98)$. Ainsi les femmes ayant un T score inférieur à $-2,5$ à la hanche après 3 ans de traitement par l'acide zolédronique, après 5 ans d'alendronate ou après 4 ans de denosumab, ont un bénéfice à poursuivre le traitement, en termes de réduction du risque de fracture vertébrale pour l'acide zolédronique et non vertébrale, pour l'alendronate et le dénosumab (99).

\subsubsection{Cible densitométrique dans l'ostéoporose}

Dans une telle stratégie la décision de durée d'une première séquence thérapeutique pourrait évoluer : à date la décision était arbitraire et reposait sur la preuve d'efficacité obtenue lors des essais contre placebo: 18 mois pour le tériparatide, 3 ans pour l'acide zolédronique et le dénosumab et 5 ans pour les autres traitements. Ce sont les durées au terme desquelles se discute la poursuite ou l'arrêt du traitement en fonction du risque fracturaire résiduel.

Une cible densitométrique serait la valeur de densité osseuse au-dessus de laquelle le risque fracturaire est réduit à un niveau admissible. Ce seuil pourrait être différent en fonction de l'âge de la patiente et du risque fracturaire prédominant :

- L'absence de perte osseuse (variation de la DMO $\leq 0,03 \mathrm{~g} / \mathrm{cm}^{2}$ ) est l'objectif minimal pour tous les patients.

- Chez les patientes dont la fragilité osseuse est révélée par une fracture sévère et caractérisée par une DMO fémorale très basse, l'objectif densitométrique du traitement est une augmentation significative de la DMO, au-dessus d'un T-score au moins égal à -2,5 voire à -2 au site fémoral (97-99). Cette stratégie peut nécessiter des changements thérapeutiques (Accord professionnel).

\subsection{Place des marqueurs du remodelage osseux}

Lorsqu'un traitement inhibant la résorption osseuse (bisphosphonates, denosumab, raloxifène et THM) est recommandé, un dosage d'un marqueur de la résorption osseuse (CTX sériques) peut être réalisé entre les $3^{\mathrm{e}}$ et $12^{\mathrm{e}}$ mois selon le traitement. La valeur mesurée doit être au minimum dans la plage des valeurs normales des femmes nonménopausées pour signifier l'effet pharmacologique du traitement. En cas de CTX sériques 
restant élevés, il est nécessaire de revoir avec la patiente les conditions de prise du traitement, et le cas échéant d'envisager un changement de traitement sous réserve des précautions d'interprétation des marqueurs: dosage à jeun, le matin, à distance d'une fracture ( $\geq 6$ mois) (Grade B).

\section{4. Évaluation morphologique du rachis}

L'évaluation morphologique du rachis à la recherche d'une fracture vertébrale (radiographies ou VFA (Vertebral Fracture Assessment)) est indiquée chez une femme ménopausée traitée s'il existe des rachialgies et/ou une perte de taille prospective $\geq 2 \mathrm{~cm}$ (90) (Accord professionnel).

\section{Tolérance des traitements}

Compte tenu de leur méthodologie, les études d'extension prolongeant les essais cliniques de phase III apportent un faible niveau de preuve d'efficacité anti-fracturaire, mais concluent à la bonne tolérance générale et osseuse des traitements prolongés. L'incidence des effets secondaires en particulier digestifs lors de traitements prolongés (jusqu'à 10 ans), par bisphosphonate n'est pas différente de celle observée dans le groupe placebo dans la phase contrôlée de l'étude.

La prise de bisphosphonates et de dénosumab est un des facteurs de risque d'ostéonécrose de la mâchoire. Dans l'ostéoporose, l'incidence des ostéonécroses de la mâchoire est très faible, comprise entre $0,001 \%$ à $0,01 \%$ patient-années selon le rapport de la Task force de l'ASBMR (100), très proche de l'incidence observée dans la population générale (cf. chapitre Suivi de l'ostéoporose).

La prise de bisphosphonates et dénosumab est un des facteurs de risque de fracture atypique fémorale. Le risque absolu de fracture atypique chez des patients recevant des bisphosphonates est très bas, compris entre 3,2 à 50 cas pour 100,000 patient-année) selon le rapport de la Task force de l'ASBMR (101). Le risque diminue à l'arrêt du traitement. D'autres facteurs de risque peuvent être associés (géométrie du fémur et du genou). Le diagnostic doit être évoqué chez une patiente traitée se plaignant de douleurs persistantes de l'aine ou des cuisses. Ces données ne remettent pas en cause la balance bénéfice-risque favorable des traitements chez les sujets à risque de fracture ostéoporotique (101-102).

L'ensemble des données publiées montre qu'il n'y pas de risque augmenté de cancer sous bisphosphonate (103). II existe d'ailleurs des données qui suggèrent un bénéfice 
oncologique (effet antitumoral) des antirésorbeurs (bisphosphonates oraux et injectables et denosumab) chez les patients ayant un cancer du sein.

Les patients doivent être prévenus d'effets secondaires rares comme les uvéites. Les patients recevant un traitement par dénosumab ont un risque d'hypocalcémie en particulier ceux ayant une carence en vitamine $D$ ou une insuffisance rénale. II est important de s'assurer que l'ostéopathie fragilisante nécessitant un traitement par denosumab ne s'intègre pas dans le cadre de complications osseuses liées à une maladie rénale chronique. Dans ces conditions il est important de vérifier la calcémie avant chaque injection.

\section{Durée du traitement}

\subsection{Durée théorique du traitement}

La durée du traitement dépend (Accord professionnel) :

- de l'âge,

- de l'évolution de la densitométrie osseuse sous traitement,

- de la tolérance osseuse et générale du traitement,

- $\quad$ de la survenue de fracture sous traitement

Les traitements ont fait la preuve de leur efficacité anti-fracturaire dans des études contrôlées de 3 à 5 ans et de 18 mois pour le tériparatide (Grade A).

Des études sont disponibles pour envisager si besoin des traitements plus prolongés : 10 ans pour l'alendronate (104-105), 7 ans pour le risédronate (106), 8 ans pour le raloxifène (107), 9 ans pour l'acide zolédronique (108) et 10 ans pour le dénosumab (109). La méthodologie de ces études ne permet pas de parler d'efficacité à long terme des traitements (en raison de l'absence de groupe contrôle) mais permet de vérifier la tolérance osseuse et extra-osseuse à long terme.

\subsection{Attitude pratique}

L'arrêt d'un traitement après une première séquence thérapeutique peut être discuté chez une patiente qui a les éléments suivants (Accord professionnel) :

- pas de fracture sous traitement,

- pas de nouveaux facteurs de risque, 
- pas de diminution significative de la $\mathrm{DMO}>0,03 \mathrm{~g} / \mathrm{cm} 2$ au rachis ou à la

hanche

- et en cas de fracture sévère, chez un patient avec un T score fémoral de fin de traitement au moins égal à $-2,5$ voire -2 .

Ces recommandations ne peuvent pas envisager tous les cas spécifiques et sont à adapter au cas par cas.

Une réévaluation après l'arrêt du traitement est recommandée après 2 ans. Ensuite le délai dépend du type de traitement. L'effet densitométrique est plus prolongé à l'arrêt de l'acide zolédronique et de l'alendronate qu'à l'arrêt des autres traitements. II n'y a en particulier pas d'effet rémanent avec le dénosumab, mais un rebond de résorption osseuse, conduisant à la perte du gain de DMO à l'arrêt.

\section{Les séquences thérapeutiques}

Certaines séquences ont été validées dans la littérature. Compte tenu de la diminution de la densité osseuse à l'arrêt du tériparatide prescrit pour 18 mois, la prescription du tériparatide doit être suivie par la prescription d'un traitement inhibant la résorption osseuse: bisphosphonates ou denosumab (110-112). À l'arrêt du dénosumab, compte tenu de la perte osseuse et du risque augmenté de fractures vertébrales multiples, certaines données montrent que les bisphosphonates sont efficaces pour prévenir la perte osseuse. Un traitement par bisphosphonate oral ou injectable pour une période de 6 à 12 mois doit dès lors être pris en relais en fin de ce traitement (113-114) (Accord professionnel).

Le relais par denosumab après bisphosphonates oraux permet d'obtenir un gain de DMO qui est supérieur à celui observé avec la poursuite des bisphosphonates (115-117). Le relais par acide zolédronique après bisphosphonates oraux ne permet pas d'augmenter la DMO par comparaison à la poursuite des bisphosphonates oraux (118).

Conflits d'intérêts :

BRIOT $\mathrm{K}$ : Interventions ponctuelles : honoraires en tant qu'expert ou orateur des laboratoires Amgen, MSD, Lilly, Pfizer

ROUX C : Interventions ponctuelles: ALEXION, AMGEN, PFIZER, UCB et Contrats de recherche pour études cliniques : ULTRAGENYX

THOMAS T: Interventions ponctuelles: Amgen, Chugaï, Expanscience, Gilead, HAC-Pharma, LCA, MSD, Novartis, Pfizer, Thuasne, UCB, Abbvie, BMS, Lilly, TEVA, Medac. 
BLAIN H: Interventions ponctuelles : honoraires en tant qu'expert ou orateur des laboratoires Lilly et Expanscience

BUCHON D : pas de conflits d'intérêts

CHAPURLAT R : Interventions ponctuelles : honoraires en tant qu'expert ou orateur des laboratoires Amgen, UCB, Radius, Lilly.

F DEBIAIS : interventions ponctuelles : honoraires en tant qu'expert ou orateur des laboratoires Amgen, Expanscience, Lilly, MSD, Novartis, Roche, Servier.

FERON JM : Interventions ponctuelles : honoraires en tant qu'expert ou orateur des laboratoires Amgen et Lilly

GAUVAIN JB : pas de conflits d'intérêts

GUGGENBUHL P : Interventions ponctuelles comme conférencier ou invitation à des congrès AMGEN, Lilly, NOVARTIS.

LEGRAND E : interventions ponctuelles : honoraires en tant qu'expert ou orateur des Amgen et Lilly

LEHR-DRYLEWICZ AM : pas de conflits d'intérêts

LESPESSAILLES E : Interventions ponctuelles: Honoraires en tant qu'expert ou orateur d'Amgen, Lilly et Intérêts indirects : Soutien financier pour des programmes de recherche ou investigateur de Amgen, Lilly, MSD, rhumatologue, Orléans

TREMOLLIERES F : Interventions ponctuelles: Honoraires en tant qu'expert ou orateur d'Expanscience, MSD, TEVA.

WERYHA Georges : interventions ponctuelles : Novartis, Procter \& Gamble, Lilly, Servier, Theramex, Daiichi Sankyo, Ipsen et Contrats de recherche pour études cliniques : Servier, Lilly, MSD, Amgen, Nycomed, Roche

CORTET B : Interventions ponctuelles : honoraires en tant qu'expert ou orateur des laboratoires Amgen, Expansience, Ferring, Lilly, Meda, Medtronic, MSD, Novartis, Roche diagnostics, UCB.

\section{Groupe de lecture}

Sauveur Bendavid (médecin généraliste, paris)

Véronique Breuil (Rhumatologue, Nice)

Laure Chapuis (Rhumatologue,

Marie Flori (Médecin généraliste

Rose Marie Javier (Rhumatologue, Strasbourg) 
Patrice Lopes (Gynécologue)

Brigitte Letombe (Gynécologue)

Philippe Merloz (Chirurgien Orthopédiste)

Yves Rolland (Gériatre)

\section{Annexe A. Matériel complémentaire}

Du matériel complémentaire (Table S1) accompagnant cet article est disponible sur ... 


\section{Références}

1. NIH Consensus Development Panel on Osteoporosis. JAMA 2001; 285: 785-95.

2. Tinetti ME, Speechley M, Ginter SF. Risk factors for falls among elderly persons living in the community.N Engl J Med. $1988 ; 319: 1701-7$.

3. Milat AJ, Watson WL, Monger C, Barr M, Giffin M, Reid M. Prevalence, circumstances and consequences of falls among community-dwelling older people : results of the 2009 NSW Falls Prevention Baseline Survey. N S W Public Health Bull. 2011;22:43-8.

4. Maravic M, Le Bihan C, Landais P, Fardellone P. Incidence and cost of osteoporotic fractures in France during 2001. A methodological approach by the national hospital database. Osteoporos Int. 2005; 16:1475-80.

5. Briot K, Maravic M, Roux C. Changes in number and incidence of hip fractures over 12 years in France. Bone. $2015 ; 81: 131-7$.

6. Thomas T, Gabach P, Buchon D et al. Évaluation de la prise en charge avant et après hospitalisation pour fracture de fragilité en France à partir des dossiers de la SNIIRAM. Congrès SFR 2015, communication O.116.

7. Solomon DH, Johnston SS, Boytsov NN, McMorrow D, Lane JM, Krohn KD. Osteoporosis medication use after hip fracture in U.S. patients between 2002 and 2011. J Bone Miner Res. 2014;29:1929-37.

8. LeBlanc ES, Hillier TA, Pedula KL, et al. Hip fracture and increased short-term but not long-term mortality in healthy older women. Arch Intern Med. 2011;171:1831-7;

9. Melton LJ 3rd, Achenbach SJ, Atkinson EJ, Therneau TM, Amin S. Long-term mortality following fractures at different skeletal sites: a population-based cohort study.Osteoporos Int. 2013; 24:1689-9.

10. Bliuc D, Nguyen ND, Milch VE, et al. Mortality risk associated with low-trauma osteoporotic fracture and subsequent fracture in men and women. JAMA 2009; 301:513-21.

11. Clinton J, Franta A, Polissar NL, Neradilek B, Mounce D, Fink HA, Schousboe JT, Matsen FA 3rd. Proximal humeral fracture as a risk factor for subsequent hip fractures. $\mathrm{J}$ Bone Joint Surg Am. 2009;91:503-11.

12. Bliuc D, Nguyen TV, Eisman JA, Center JR. The impact of nonhip nonvertebral fractures in elderly women and men. J Clin Endocrinol Metab. 2014; 99:415-23.

13. Cuddihy MT, Gabriel SE, Crowson CS, O'Fallon WM, Melton LJ 3rd. Forearm fractures as predictors of subsequent osteoporotic fractures. Osteoporos Int. 1999;9:469-75.

14.Lindsay R, Silverman SL, Cooper C, et al. Risk of new vertebral fracture in the year following a fracture. JAMA. 2001;285:320-3. 
15. van Geel TA, van Helden S, Geusens PP, Winkens B, Dinant GJ. Clinical subsequent fractures cluster in time after first fractures. Ann Rheum Dis. 2009; 68:99-102.

16. van Geel TA, Nguyen ND, Geusens PP, et al. Development of a simple prognostic nomogram for individualising 5-year and 10-year absolute risks of fracture : a populationbased prospective study among postmenopausal women. Ann Rheum Dis. 2011;70:92-7.

17. Bonafede M, Shi N, Barron R, Li X, Crittenden DB, Chandler D. Predicting imminent risk for fracture in patients aged 50 or older with osteoporosis using US claims data. Arch Osteoporos. 2016;11:26.

18. Huntjens KM, van Geel TA, van Helden S, et al. The role of the combination of bone and fall related risk factors on short-term subsequent fracture risk and mortality. BMC Musculoskelet Disord. 2013;14:121.

19. Amouzougan A, Lafaie L, Marotte H, Dẻnariẻ D, Thomas T. High prevalence of dementia in women with osteoporosis. Joint Bone Spine 2017; 84: 611-614

20. Laroche $M$, Pécourneau V, Blain $H$, Breuil V, the GRIO scientific committee. Osteoporosis and ischemic cardiovascular disease Joint Bone Spine 2017; 84: 427-32.

21. Black DM, Arden NK, Palermo L, Pearson J, Cummings SR. Prevalent vertebral deformities predict hip fractures and new vertebral deformities but nop wrist fractures. J Bone Miner Res 1999; 5: 821-8.

22. Klotzbuecher CM, Ross PD, Landsman PB, Abbott TA, Berger M. Patients with prior fractures have an increased risk of future fractures: a summary of the literature and statistical synthesis. J Bone Miner Res 2000; 15: 721-39.

23. Giangregorio LM, Leslie WD; Manitoba Bone Density Program. Time since prior fracture is a risk modifier for 10-year osteoporotic fractures.J Bone Miner Res. 2010; 25:1400-5.

24. World Health Organization (WHO). Assessment of fracture risk and its application to screening for postmenopausal osteoporosis : report of a WHO study group. WHO Technical Report Series n॰843. WHO, Genève, Suisse, 1994; 1-29.

25. Lewiecki EM, Watts NB, McClung MR, et al. Official position of the International Society for Clinical Densitometry. J Clin Endocrinol Metab 2004; 89:3651-5.

26. Kanis JA, McCloskey EV, Johansson H, Oden A, Melton LJ 3rd, Khaltaev N. A reference standard for the description of osteoporosis. Bone 2008;42:467-75.

27. Paggiosi MA, Gluer CC, Roux C, et al. International variation in proximal femur bone mineral density. Osteoporos Int 2011; 22: 721-9.

28. Marshall $\mathrm{D}$, Johnell $\mathrm{O}$, Wedel $\mathrm{H}$. Metanalysis of how well measures of bone mineral density predict occurrence of osteoporotic fractures. BMJ 1996; 312: 1254-9. 
29. Fujiwara S, Kasagi F, Masunari N, Naito K, Suzuki G, Fukunaga M. Fracture prediction from bone mineral density in Japanese men and women. J Bone Miner Res. 2003;18:154753.

30. Sornay-Rendu E, Munoz F, Garnero P, Duboeuf F, Delmas PD. Identification of osteopenic women at high risk of fracture : the OFELY study. J Bone Miner Res 2005; 20: 1813-9.

31. Johnell O, Kanis JA, Oden A, et al. Predictive value of BMD for hip and other fractures. J Bone Miner Res. 2005; 20: 1185-94.

32. Bates DW, Black DM, Cummings SR Clinical use of bone densitometry: clinical applications. JAMA. 2002;288:1898-900.

33. Cummings SR, Black DM, Nevitt MC, et al. Bone density at various sites for prediction of hip fractures. The Study of Osteoporotic Fractures Research Group. Lancet. 1993;341:72-5.

34. Schuit Sc, van der Klift M, Weel AE, de Laet CE, Burger $H$, Seeman E. Fracture incidence and association with bone mineral density in elderly men and women: the Rotterdam Study. Bone 2004; 34: 195-202.

35 Siris ES, Chen YT, Abbott TA, et al. Bone mineral density threshold for pharmacological intervention to prevent fractures. Arch Intern Med. 2004;164:1108-12.

36. Wainwright SA, Marshall LM, Ensrud KE, et al. Hip fracture in women without osteoporosis. J Clin Endocrinol Metab. 2005;90:2787-93.

37. Schwartz AV, Vittinghoff E, Bauer DC, et al. Association of BMD and FRAX score with risk of fracture in older adults with type 2 diabetes. JAMA. 2011;305:2184-92.

38. Premaor MO, Ensrud K, Lui L, Parker RA, Cauley J, Hillier TA, Cummings S, Compston JE; Study of Osteoporotic Fractures Risk factors for nonvertebral fracture in obese older women. J Clin Endocrinol Metab. 2011;96:2414-21.

39. Premaor MO, Pilbrow L, Tonkin C, Parker RA, Compston J. Obesity and fractures in postmenopausal women.J Bone Miner Res. 2010;25:292-7.

40. Yamamoto M, Yamaguchi T, Yamauchi M, Kaji H, Sugimoto T. Diabetic patients have an increased risk of vertebral fractures independent of BMD or diabetic complications. $J$ Bone Miner Res. 2009;24:702-9

41. Gnant M, Mlineritsch B, Luschin-Ebengreuth G, e al.Adjuvant endocrine therapy plus zoledronic acid in premenopausal women with early-stage breast cancer : 5-year follow-up of the ABCSG-12 bone-mineral density substudy. Lancet 2015;386: 433-43.

42. Dargent-Molina P, Favier F, Grandjean $H$, et al. Fall-related factors and risk of hip fracture : the EPIDOS prospective study. Lancet. 1996; 348:145-9.

43. American Geriatrics Society (2010) British geriatrics Society clinical practice guideline : prevention of falls in older persons.American Geriatrics Society, New York. 
http ://www.american geriatrics.org/healthcareprofessionals/clinicalpractice/clinicalguidelines recommendations/2010.

44. Blain H, Masud T, Dargent-Molina $P$, et al. A Comprehensive Fracture Prevention Strategy in Older Adults: The European Union Geriatric Medicine Society (EUGMS) Statement. J Nutr Health Aging. 2016;20:647-52.

45. Kanis JA, Johnell O, Oden A, Johansson H, McCloskey E. FRAX® and the assessment of fracture probability in men and women from the UK. Osteoporos Int 2008; 19: 385-97.

46. Briot K, Cortet B, Thomas $\mathrm{T}$, et al.2012 update of French guidelines for the pharmacological treatment of postmenopausal osteoporosis.Joint Bone Spine. 2012;79:30413.

47. Briot K, Paternotte S, Kolta S, et al. FRAX®: prediction of major osteoporotic fractures in women from the general population : the OPUS study. PLoS One. 2013; 8:e83436.

48. Sornay-Rendu E, Munoz F, Delmas PD, Chapurlat RD. The FRAX tool in French women: How well does it describe the real incidence of fracture in the OFELY cohort? J Bone Miner Res. 2010; 25:2101-7.

49. Trémollieres FA, Pouillès JM, Drewniak N, Laparra J, Ribot CA, Dargent-Molina P. Fracture risk prediction using BMD and clinical risk factors in early postmenopausal women. Sensitivity of the WHO FRAX tool. J Bone Miner Res. 2010;25:1002-9.

50. Couris CM, Chapurlat RD, Kanis JA, Johansson H, Delmas PD, Schott AM. FRAX® probabilities and risk of major osteoporotic fracture in France. Osteoporos Int 2012; 23:23217.

51. Bousson V, Bergot C, Sutter B, et al. Trabecular Bone Score : Where are we now? Joint Bone Spine. 2015;82:320-5.

53. Leslie WD, Johansson H, Kanis JA, Lamy O, Oden A, McCloskey EV, Hans D. Lumbar spine texture enhances 10-year fracture probability assessment. Osteoporos Int. 2014;25:2271-7.

54. Leslie WD, Aubry-Rozier B, Lix LM, Morin SN, Majumdar S5, Hans D; Spine bone texture assessed by trabecular bone score (TBS) predicts osteoporotic fractures in men: the Manitoba Bone Density Program. Bone. 2014. 67 :10-4.

55. McCloskey EV, Odén A, Harvey NC, et al. A Meta-Analysis of Trabecular Bone Score Osteoporos Int 2015;22: 391-420.

56. Vasikaran $S$, Eastell $R$, Bruyère $O$, et al. Fracture Risk Prediction and Its Relationship to FRAX. Markers of bone turnover for the prediction of fracture risk and monitoring of osteoporosis treatment: a need for international reference standards. Osteoporos int ;22:391-420. 
58. Cauley JA, Robbins J, Chen Z, et al. Effects of estrogen plus progestin on risk of fracture and bone mineral density. The women's Health Initiative randomized trial. JAMA 2003; 290 : 1729-38.

59. Ettinger B, Black DM, Mitlakj BM, et al. Reduction of vertebral fracture risk in postmenopausal women with osteoporosis treated with raloxifEne. Results from a 3 years randomized clinical trial. JAMA 1999; 282: 637-45.

60. Black DM, Cummings SR, Karpf DB et al. Randomised trial of effect of alendronate on risk of fracture in women with existing vertebral fractures. Fracture intervention Trial Research Group. Lancet 1996 ; 348 : 1535-41.

61. Cummings SR, Black DM, Thompson DE et al. Effect of alendronate on risk of fracture in women with low bone density but without vertebral fractures: results from the Fracture Intervention trial. JAMA 1998; 280: 2077-82.

62. Cranney A, Wells G, Willan A, et al. Meta-analysis of alendronate for the treatment of postmenopausal women. Endocrine Reviews 2002; 23: 508-16.

63. Wells GA, Cranney A, Peterson J, et al. Alendronate for the primary and secondary prevention of osteoporotic fractures in postmenopausal women. Cochrane Database Syst Rev. 2008;(1):CD001155.

64. Harris ST, Watts NB, Genant HK,et al. Effect of risedronate treatment on vertebral and nonvertebral fractures in women with postmenopausal osteoporosis. A randomized controlled trial. JAMA, 1999; 282: 1344-52.

65. Reginster JY, Minne HW, Sorensen $\mathrm{OH}$ et al. Randomized trial of the effects of risedronate on vertebral fractures in women with established postmenopausal osteoporosis. Osteoporosis Int 2000; $11: 83-91$.

66. Wells G, Cranney A, Peterson J, et al. Risedronate for the primary and secondary prevention of osteoporotic fractures in postmenopausal women. Cochrane Database Syst Rev. 2008;(1):CD004523.

67. Boonen S, McClung MR, Eastell R, El-Hajj Fuleihan, Barton IP, Delmas P. Safety and efficacy of risedronate in reducing fracture risk in osteoporotic women aged 80 and older : implications for the use of antiresorptive agents in the old and oldest old. J Am Geriatr Soc. 2004; 52: 1832-9.

68. Black DM, Delmas PD, Eastell R, et al. Once-yearly zoledronic acid for treatment of postmenopausal osteoporosis. N Engl J Med. 2007; 356:1809-22.

69 Lyles KW, Colón-Emeric CS, Magaziner JS, et al. Zoledronic Acid in Reducing Clinical Fracture and Mortality after Hip Fracture.N Engl J Med. 2007; 357:nihpa40967.

70. Boonen S, Black DM, Colón-Emeric CS, et al. Efficacy and safety of a once-yearly intravenous zoledronic acid $5 \mathrm{mg}$ for fracture prevention in elderly postmenopausal women with osteoporosis aged 75 and older. J Am Geriatr Soc. 2010; 58: 2 92-9. 
71. Neer RM, Arnaud CD, Zanchetta JR, et al. Effect of parathyroid hormone (1-34) on fractures and bone mineral density in postmenopausal women with osteoporosis. N Engl J Med 2001; 344: 1434-41.

72. Cummings SR, San Martin J, McClung MR, et al. Denosumab for prevention of fractures in postmenopausal women with osteoporosis. N Engl J Med. 2009; 361:756-65.

73. Karpf DB, Shapiro DR, Seeman E et al. Prevention of non vertebral fractures by alendronate. A meta-analysis. Alendronate Osteoporosis Treatment Study groups. JAMA 1997; 277: 1159-64.

74. Clung MR, Gensens P, Miller PD, et al. Effect of risedronate on the risk of hip fracture in elderly women. N Engl J Med 2001; 344: 333-340.

75. Papapoulos SE, Quandt Sa, Liberman UA et al. Metaanalysis of the efficacy of alendronate for the prevention of hip fractures in postmenopausal women. Osteoporos int 2005; 16: 468-74.

76. Ganda K, Puech M, Chen JS, et al. Models of care for the secondary prevention of osteoporotic fractures : a systematic review and meta-analysis. Osteoporos Int. 2013;24:393406.

77. .Huntjens KM, van Geel TA, van den Bergh JP, et al. Fracture liaison service : impact on subsequent nonv ertebral fracture incidence and mortality. J Bone Joint Surg Am. 2014;96:e29.

78. Blain $\mathrm{H}$, Rolland $\mathrm{Y}$, Beauchet $\mathrm{O}$, et al. Usefulness of bone density measurement in fallers.Joint Bone Spine. 2014;81:403-8.

79. Gillespie LD, Robertson MC, Gillespie WJ, et al. Interventions for preventing falls in older people living in the community. Cochrane Database Syst Rev. 2012 ;(9):CD007146.

80. El-Khoury F, Cassou B, Charles MA, Dargent-Molina P. The effect of fall prevention exercise programmes on fall induced injuries in community dwelling older adults : systematic review and meta-analysis of randomised controlled trials. BMJ. 2013;347:f6234.

81. El-Khoury F, Cassou B, Latouche A, Aegerter P, Charles MA, Dargent-Molina P. Effectiveness of two year balance training programme on prevention of fall induced injuries in at risk women aged 75-85 living in community : Ossébo randomised controlled trial. BMJ. 2015;351:h3830.

82. Gill TM, Pahor M, Guralnik JM, et al. Effect of structured physical activity on prevention of serious fall injuries in adults aged 70-89: randomized clinical trial (LIFE Study). BMJ. 2016;352:i245.

83. Bolland MJ, Barber PA, Doughty RN, et al. Vascular events in healthy older women receiving calcium supplementation : randomised controlled trial. BMJ 2008; 336:262-6. 
84. Bolland MJ, Avenell A, Baron JA, Grey A, MacLennan GS, Gamble GD, Reid IR. Effect of calcium supplements on risk of myocardial infarction and cardiovascular events : metaanalysis. BMJ 2010; 341:c3691.

85. Pentti K, Tuppurainen MT, Honkanen R, et al. Use of calcium supplementation and the risk of coronary heart disease in 52-62-year-old women: the Kuopio Osteoporosis Risk Factor and Prevention Study. Maturitas. 2009; 63:73-8.

86. Bolland MJ, Grey A, Avenell A, Gamble GD, Reid IR. Calcium supplements with or without vitamin $D$ and risk of cardiovascular events : reanalysis of the Women's Health Initiative limited access dataset and meta-analysis. BMJ. 2011;342:d2040.

87. Sanders KM, Stuart AL, Williamson EJ, et al. Annual high-dose oral vitamin $D$ and falls and fractures in older women : a randomized controlled trial. JAMA 2010; 303:1815-22.

88. Richardson K, Bennett K, Kenny RA. Polypharmacy including falls risk-increasing medications and subsequent falls in community-dwelling middle-aged and older adults. Age Ageing. 2015;44:90-6

89. Teng Z, Zhu Y, Wu F, Zhu Y, Zhang X, Zhang C, Wang S, Zhang L. Opioids contribute to fracture risk : a meta-analysis of 8 cohort studies. PLoS One. $2015 ; 10(6): e 0128232$.

90. Siminoski K, Jiang G, Adachi JD, Hanley et al. The accuracy of height loss during prospective monitoring for detection of incident vertebral fractures. Osteoporos Int 2005; 16:403-410.

91. Siminoski K, Warshawski RS, Jen H, Lee K. The accuracy of historical height loss for the detection of vertebral fractures in postmenopausal women. Osteoporos Int 2006; 17: 290-6.

92. Briot K, Legrand E, Pouchain D, Monnier S, Roux C. Accuracy of patient-reported height loss and risk factors for height loss among postmenopausal women. CMAJ. 2010; 6; 182:558-62.

94. Modi A, Sen S, Adachi JD, et al. Association of gastrointestinal events with quality of life and treatment satisfaction in osteoporosis patients: results from the Medication Use Patterns, 93 Satisfaction, and Inadequate Control of Osteoporosis Study (MUSIC OS). Osteoporos Int. 2017 ;28(10):2867-76.

94 Ravaud P, Reny JL, Giraudeau B, Porcher R, Dougados M, Roux C. Individual smallest detectable difference in bone mineral density measurements. $J$ Bone Miner Res. 1999;14:1449-56.

95. Jacques RM, Boonen S, Cosman F, Reid IR, Bauer DC, Black DM, Eastell R. Relationship of changes in total hip bone mineral density to vertebral and nonvertebral fracture risk in women with postmenopausal osteoporosis treated with once-yearly zoledronic acid $5 \mathrm{mg}$ : the HORIZON-Pivotal Fracture Trial (PFT). J Bone Miner Res. 2012;27:1627-34. 
96. Austin M, Yang YC, Vittinghoff E, et al. Relationship between bone mineral density changes with denosumab treatment and risk reduction for vertebral and nonvertebral fractures.J Bone Miner Res. 2012;27:687-93.

97. Bauer DC, Schwartz A, Palermo L, et al.Fracture prediction after discontinuation of 4 to 5 years of alendronate therapy : the FLEX study. JAMA Intern Med. 2014;174:1126-34.

98. Cosman F, Cauley JA, Eastell R, et al. Reassessment of fracture risk in women after 3 years of treatment with zoledronic acid: when is it reasonable to discontinue treatment? $\mathrm{J}$ Clin Endocrinol Metab. 2014;99:4546-54

99. Ferrari S, Adachi JD, Lippuner K, et al. Further reductions in nonvertebral fracture rate with long-term denosumab treatment in the FREEDOM open-label extension and influence of hip bone mineral density after 3 years. Osteoporos Int. 2015;26:2763-71.

100. Khan AA, Morrison A, Hanley DA, et al. Diagnosis and management of osteonecrosis of the jaw : a systematic review and international consensus. J Bone Miner Res. 2015;30:3-23.

101. Shane E, Burr D, Abrahamsen B, et al. Atypical subtrochanteric and diaphyseal femoral fractures: second report of a task force of the American Society for Bone and Mineral Research. J Bone Miner Res. 2014. 29 :1-23.

102. Gedmintas L, Solomon DH, Kim SC. Bisphosphonates and risk of subtrochanteric, femoral shaft, and atypical femur fracture : a systematic review and meta-analysis. J Bone Miner Res. 2013;28:1729-37.

103. Cardwell CR, Abnet CC, Cantwell MM, Murray LJ. Exposure to oral bisphosphonates and risk of esophageal cancer. JAMA. 2010;304:657-63.

104 Bone HG, Hosking D, Devogelaer JP, et al. Ten years' experience with alendronate for osteoporosis in postmenopausal women. N Engl J Med. 2004;350:1189-99.

105. Black DM, Schwartz AV, Ensrud KE, Cauley JA, Levis S, Quandt SA, Satterfield S, Wallace RB, Bauer DC, Palermo L, Wehren LE, Lombardi A, Santora AC, Cummings SR; FLEX Research Group. Effects of continuing or stopping alendronate after 5 years of treatment : the Fracture Intervention Trial Long-term Extension (FLEX) : a randomized trial. JAMA. 2006 ;296:2927-38.

106. Mellström DD, Sörensen OH, Goemaere S, Roux C, Johnson TD, Chines AA. Seven years of treatment with risedronate in women with postmenopausal osteoporosis. Calcif Tissue Int. 2004;75:462-8.

107. Martino S, Cauley JA, Barrett-Connor E, et al. Continuing outcomes relevant to Evista: breast cancer incidence in postmenopausal osteoporotic women in a randomized trial of raloxifene. J Natl Cancer Inst 1;96:1751-61.

108. Black DM, Reid IR, Cauley JA, et al. The effect of 6 versus 9 years of zoledronic acid treatment in osteoporosis : a randomized second extension to the HORIZON-Pivotal Fracture Trial (PFT).J Bone Miner Res. 2015;30:934-44. 
109. Bone HG, Wagman RB, Brandi ML, et al. 10 years of denosumab treatment in postmenopausal women with osteoporosis : results from the phase 3 randomised FREEDOM trial and open-label extension. Lancet Diabetes Endocrinol. 2017; S2213-8587 : 30138-9.

110 Lindsay R, Scheele WH, Neer R, et al. Sustained vertebral fracture risk reduction after withdrawal of teriparatide in postmenopausal women with osteoporosis. Arch Intern Med 2004; 164: 2024-30.

111. Prince R, Sipos A, Hossain A, Syversen U, et al. Sustained non vertebral fragility fracture risk reduction after discontinuation of teriparatide treatment. J Bone Miner Res 2005; 20:1507-13.

112. Leder BZ, Tsai JN, Uihlein AV, Wallace PM, Lee H, Neer RM, Burnett-Bowie SA. Denosumab and teriparatide transitions in postmenopausal osteoporosis (the DATA-Switch study) : extension of a randomised controlled trial. Lancet. 2015;386:1147-55.

113. Freemantle N, Satram-Hoang S, Tang ET, et al. Final results of the DAPS (Denosumab Adherence Preference Satisfaction) study : a 24-month, randomized, crossover comparison with alendronate in postmenopausal women. Osteoporos Int. 2012;23:317-26.

114. Cummings SR, Ferrari S, Eastell R, et al. Vertebral Fractures After Discontinuation of Denosumab: A Post Hoc Analysis of the Randomized Placebo-Controlled FREEDOM Trial and Its Extension.J Bone Miner Res. 2018;33:190-8.

115. Kendler DL, Roux C, Benhamou CL, et al. Effects of denosumab on bone mineral density and bone turnover in postmenopausal women transitioning from alendronate therapy. J Bone Miner Res. 2010 ;25:72-81.

116. Roux C, Hofbauer LC, Ho PR, et al. Denosumab compared with risedronate in postmenopausal women suboptimally adherent to alendronate therapy : efficacy and safety results from a randomized open-label study. Bone 2014;58:48-54.

117. Miller PD, Pannacciulli N, Brown JP, et al. Denosumab or Zoledronic Acid in Postmenopausal Women With Osteoporosis Previously Treated With Oral Bisphosphonates.J Clin Endocrinol Metab. 2016;101:3163-70.

118. McClung M, Recker R, Miller $P$, et al. Intravenous zoledronic acid $5 \mathrm{mg}$ in the treatment of postmenopausal women with low bone density previously treated with alendronate. Bone. 2007;41:122-8. 
Encadré 1 : Indications remboursées en France de l'absorptiométrie biphotonique à rayons X (Journal Officiel du 30 juin 2006 "Décision du 29 juin 2006 de l'Union nationale des caisses d'assurance maladie relative à la liste des actes et prestations pris en charge par l'assurance maladie)

\section{Pour un premier examen :}

\section{Dans la population générale, quels que soient l'âge et le sexe :}

1. En cas de signes d'ostéoporose : découverte ou confirmation radiologique d'une fracture vertébrale (déformation du corps vertébral) sans contexte traumatique ni tumoral évident ; antécédent personnel de fracture périphérique survenue sans traumatisme majeur (sont exclues de ce cadre les fractures du crâne, des orteils, des doigts, du rachis cervical) ;

2. En cas de pathologie ou traitement potentiellement inducteur d'ostéoporose : lors d'une corticothérapie systémique (de préférence au début) prescrite pour une durée d'au moins 3 mois consécutifs, à une dose $>7,5 \mathrm{mg} / \mathrm{jour}$ d'équivalent prednisone ;

3. Antécédent documenté de pathologie ou de traitement potentiellement inducteur d'ostéoporose : hypogonadisme prolongé (incluant l'androgénoprivation chirurgicale [orchidectomie] ou médicamenteuse [traitement prolongé par un analogue de la Gn-Rh]), hyperthyroïdie évolutive non traitée, hypercorticisme, hyperparathyroïdie primitive et ostéogenèse imparfaite ;

Chez la femme ménopausée (y compris pour les femmes sous traitement hormonal de la ménopause à des doses utilisées inférieures aux doses recommandées pour la protection osseuse), indications supplémentaires (par rapport à la population générale) :

1. Antécédent de fracture du col fémoral sans traumatisme majeur chez un parent au 1er degré;

2. Indice de masse corporelle $<19 \mathrm{~kg} / \mathrm{m}^{2}$;

3. Ménopause avant 40 ans, quelle qu'en soit la cause ;

4. Antécédent de prise de corticoïdes d'une durée d'au moins 3 mois consécutifs, à une dose $>7,5 \mathrm{mg} /$ jour équivalent prednisone.

Pour un second examen :

- A l'arrêt du traitement anti-ostéoporotique, en dehors de l'arrêt précoce pour effet indésirable, chez la femme ménopausée ;

- Chez la femme ménopausée sans fracture, lorsqu'un traitement n'a pas été mis en route après une première ostéodensitométrie montrant une valeur normale ou une ostéopénie, une deuxième ostéodensitométrie peut être proposée 3 à 5 ans après la réalisation de la première en fonction de l'apparition de nouveaux facteurs de risque. 
Tableau $^{\circ} 1$ : Facteurs de risque de chutes

\begin{tabular}{|l|l|}
\hline \multicolumn{1}{|c|}{ Facteurs intrinsèques } & \multicolumn{1}{c|}{ Facteurs extrinsèques } \\
\hline Age (plus de 80 ans) & Consommation d'alcool \\
Antécédent de chute dans l'année précédente & Sédentarité \\
Troubles locomoteurs et neuromusculaires : & Malnutrition \\
- diminution de la force musculaire des & Facteurs environnementaux : \\
membres inférieurs & - habitat mal adapté (escaliers, tapis) \\
- préhension manuelle réduite & - utilisation ou non utilisation d'une aide à \\
- difficultés à la marche & la marche \\
- troubles de l'équilibre & - environnement public (trottoirs \\
Baisse de l'acuité visuelle & irréguliers, surfaces glissantes) \\
Baisse de l'audition & - mauvaise utilisation ou non utilisation \\
Prise de psychotropes & d'une canne \\
Polymédication (au-delà de 4) & Facteurs socioéconomiques : éducation, \\
Pathologies spécifiques : & revenu, logement, intégration sociale \\
- maladie de Parkinson & \\
- démences & \\
- dépression & \\
- séquelles d'accident vasculaire cérébral & \\
- carence en vitamine D & \\
\hline
\end{tabular}


Encadré $^{\circ} 2$ : Facteurs de risque de fracture

\begin{tabular}{|c|c|}
\hline & $\begin{array}{l}\text { âge* } \\
\text { origine caucasienne } \\
\text { ménopause avant } 40 \text { ans } \\
\text { aménorrhée primaire ou secondaire } \\
\text { antécédent familial de fracture par fragilité osseuse* } \\
\text { antécédent personnel de fracture* } \\
\text { faible poids* } \\
\text { troubles de l'acuité visuelle* } \\
\text { troubles neuromusculaires* } \\
\text { immobilisation très prolongée* } \\
\text { tabagisme* } \\
\text { corticothérapie* } \\
\text { faible apport calcique } \\
\text { carence en vitamine D } \\
\text { consommation excessive d'alcool }\end{array}$ \\
\hline
\end{tabular}

* facteurs de risque de fracture ostéoporotique indépendant de la DMO 
Tableau $^{\circ} 2$ : Effet des traitements sur le risque de fracture vertébrale

\begin{tabular}{|c|c|c|c|c|}
\hline Traitement & Etude & Durée & Risque Relatif & Commentaires \\
\hline THM & WHI [1] & 5 ans & Toutes fractures (y compris FV) : 0,76 $(0,69-0,85)$ & \\
\hline Raloxifène & MORE (Delmas) & $\begin{array}{l}3 \text { ans } \\
4 \text { ans }\end{array}$ & $\begin{array}{l}0,7(0,5-0,8) \\
0,64(0,53-0,76)\end{array}$ & Réduction de la $4^{\mathrm{ème}}$ année comparable aux 3 premières années \\
\hline Alendronate & $\begin{array}{l}\text { FIT } 1 \text { (Black) } \\
\text { FIT } 2 \text { (Cummings) } \\
\text { Méta-analyse (Cranney) } \\
\text { Méta-analyse (Wells) }\end{array}$ & $\begin{array}{l}3 \text { ans } \\
4 \text { ans } \\
2 \text {-3ans } \\
\geq 1 \text { an }\end{array}$ & $\begin{array}{l}0,53(0,41-0,68) \\
0,45(0,27-0,72) \\
0,56(0,39-0,80) \\
0,52(0,43-0,65) \\
0,55(0,38-0,80) \\
0,55(0,43-0,69)\end{array}$ & $\begin{array}{l}\text { Morphométriques } \\
\text { Fractures vertébrales cliniques } \\
\text { Critère secondaire } \\
\text { Prévention primaire } \\
\text { Prévention secondaire }\end{array}$ \\
\hline Risedronate & $\begin{array}{l}\text { Vert NA (Harris) } \\
\text { Vert MN (Reginster) } \\
\text { Analyse poolée VERT-NA et MN } \\
\text { Méta-analyse (Wells) } \\
\text { Méta-analyse (Boonen) }\end{array}$ & $\begin{array}{l}3 \text { ans } \\
3 \text { ans } \\
1 \text { an } \\
\geq 1 \text { an } \\
3 \text { ans }\end{array}$ & $\begin{array}{l}0,59(0,42-0,82) \\
0,51((0,36-0,73) \\
0,38(0,25-0,56) \\
0,61(0,50-0,76) \\
0,19(0,09-0,4)\end{array}$ & $\begin{array}{l}\text { Femmes à risque ( } \geq 1 \mathrm{FV} \text { et } \mathrm{T} \leq-2,5) \\
\text { Prévention secondaire } \\
\text { Analyse post-hoc chez sujets } \geq 80 \text { ans }\end{array}$ \\
\hline Acide zolédronique & $\begin{array}{l}\text { HORIZON PFT (Black) } \\
\text { HORIZON RFT (Lyles) } \\
\text { Analyse poolée sujets } \geq 75 \text { ans }\end{array}$ & $\begin{array}{l}3 \text { ans } \\
3 \text { ans } \\
3 \text { ans }\end{array}$ & $\begin{array}{l}0,3(0,24-0,38) \\
0,54(0,32-0,92) \\
0,34(0,21-0,55)\end{array}$ & $\begin{array}{l}\text { Chez sujets avec fracture de l'ESF } \\
\text { Analyse post-hoc (FV cliniques) }\end{array}$ \\
\hline Tériparatide & Essai PFT (Neer) & 18 mois & $0,35(0,45-0,78)$ & \\
\hline Dénosumab & FREEDOM (Cummings) & 3 ans & $0,32(0,26-0,41)$ & \\
\hline
\end{tabular}


Tableau $^{\circ} 3$ : Effet des traitements sur le risque de fracture non vertébrale

\begin{tabular}{|c|c|c|c|c|}
\hline Traitement & Etude & Durée & Risque Relatif & Commentaires \\
\hline THM & WHI [Cauley] & 5 ans & Toutes fractures (y compris FV) : 0,76 $(0,69-0,85)$ & Hanche $0,66(0,45-0,98)$ \\
\hline Raloxifène & MORE (Ettinger) & 3 ans & $0,92(0,8-1,1)(\mathrm{NS})$ & Post-HOC dans un groupe sélectionné à haut risque $0,53(0,29-0,99)$ \\
\hline Alendronate & $\begin{array}{l}\text { FIT } 1 \text { (Black) } \\
\text { FIT } 2 \text { (Cummings) } \\
\text { Méta-analyse (Karpf) } \\
\text { Méta-analyse (Cranney) } \\
\text { Méta-analyse (Wells) }\end{array}$ & $\begin{array}{l}3 \text { ans } \\
4 \text { ans } \\
3 \text { ans } \\
3 \text { ans } \\
\geq 1 \text { an }\end{array}$ & $\begin{array}{l}0,80(0,63-1,01) \\
0,86(0,73-1,01) \\
0,71(0,50-0,997) \\
0,51(0,38-0,69) \\
0,77(0,64-0,92)\end{array}$ & $\begin{array}{l}\text { Hanche : } 0,49(0,23-0,99) \\
\text { Poignet : } 0,52(0,31-0,87) \\
\text { Fracture de hanche : RR=0,47 }(0,26-0,85)\end{array}$ \\
\hline Risedronate & $\begin{array}{l}\text { Vert NA (Harris) } \\
\text { Vert MN (Reginster) } \\
\text { Hip Study (McClung) } \\
\text { Méta-analyse (Wells) } \\
\text { Méta-analyse (Boonen)] }\end{array}$ & $\begin{array}{l}3 \text { ans } \\
3 \text { ans } \\
3 \text { ans } \\
\geq 1 \text { an } \\
3 \text { ans }\end{array}$ & $\begin{array}{l}0,61(0,39-0,94) \\
0,67(0,44-1,04) \\
0,7(0,6-0,9) \\
0,80(0,72-0,90) \\
\text { NS }\end{array}$ & $\begin{array}{l}\text { Patientes avec au moins une fracture vertébrale. } \\
\text { Patientes avec au moins deux fractures vertébrales. } \\
\text { Seulement chez les femmes ostéoporotiques de } 70-79 \text { ans }(0,4(0,6-0,9) \\
\text { Fracture de hanche : RR }=0,74(0,59-0,94) \\
\text { Analyse post-hoc (sujets } \geq 80 \text { ans) }\end{array}$ \\
\hline Acide zolédronique & $\begin{array}{l}\text { HORIZON PFT (Black) } \\
\text { HORIZON RFT (Lyles) } \\
\text { Analyse poolée sujets } \geq 75 \text { ans (Boonen) }\end{array}$ & $\begin{array}{l}3 \text { ans } \\
3 \text { ans } \\
3 \text { ans }\end{array}$ & $\begin{array}{l}0.75(0,64-0,87) \\
0,73(0,55-0,98) \\
0,73(0,60-0,90)\end{array}$ & $\begin{array}{l}\text { Fracture de hanche : } 0,59(0,42-0,83) \\
\text { Chez sujets avec fracture de l'ESF ; Fracture de hanche : } 0,70(0,41-1,19) \\
\text { Analyse post-hoc }\end{array}$ \\
\hline Tériparatide & Essai PFT (Neer) & 18 mois & $0,47(0,25-0,88)$ & \\
\hline Deosumab & FREEDOM (Cummings) & 3 ans & $0,80(0,67-0,95)$ & Hanche : RR=0,60 $(0,37-0,97)$ \\
\hline
\end{tabular}


Encadré 3 : Exemples de stratégies thérapeutiques thérapeutiques dans différentes situations cliniques

\section{Exemples de stratégies thérapeutiques dans différentes situations cliniques}

1. Chez les patients ayant eu une fracture de l'ESF, l'acide zolédronique est à considérer en première intention car il est le seul traitement ayant démontré un effet anti-fracturaire dans une telle population (Grade A).

2. Chez les patients ayant deux fractures vertébrales prévalentes : le tériparatide peut être prescrit en première intention au moment du diagnostic en l'absence de contre-indication (Grade $A$ ).

3. Chez des femmes de moins de 70 ans avec une ostéoporose nécessitant un traitement, le raloxifène est à considérer si le risque de fracture non vertébrale est faible, défini par l'absence des critères suivants: T-score bas à la hanche, facteurs de risque de chute et antécédent de fracture non vertébrale (Grade A).

4 Chez une femme âgée de moins de 60 ans ayant des troubles climatériques et une ostéoporose sans fracture sévère, un THM peut être envisagé (Grade A).

5. En cas de fracture sévère avec une DMO très abaissée $(T \leq-3)$ des traitements injectables peuvent être utilisés pour atteindre une cible densitométrique ( $T>-2.5$ à -2 à la hanche) en fin de séquence : acide zolédronique, dénosumab (en cas d'échec ou intolérance aux bisphosphonates), ou séquence thérapeutique tériparatide (remboursé si présence d'au moins 2 fractures vertébrales) suivi d'un antirésorbeur (Accord professionnel). 
Tableau 4 : suivi des traitements anti-ostéoporotiques

\begin{tabular}{|c|c|c|c|c|c|c|c|c|}
\hline Traitements & $\begin{array}{l}\text { Absence de } \\
\text { fracture }\end{array}$ & $\begin{array}{l}\text { Nouveaux } \\
\text { facteurs } \\
\text { de risque }\end{array}$ & Taille & Adhésion & Tolérance & Ostéodensitométrie & $\begin{array}{l}\text { Marqueurs } \\
\text { du } \\
\text { remodelage } \\
\text { osseux }\end{array}$ & $\begin{array}{l}\text { Évaluation } \\
\text { morphologique } \\
\text { du rachis }\end{array}$ \\
\hline Acide alendronique & + & + & $\begin{array}{l}1 \\
\text { fois/an }\end{array}$ & + & + & 2 à 3 ans & $\begin{array}{l}3 \text { à } 12 \text { mois } \\
\text { après le } \\
\text { début du } \\
\text { traitement }\end{array}$ & $\begin{array}{l}\text { Si perte de } \\
\text { taille/rachialgies }\end{array}$ \\
\hline Acide risédronique & + & + & $\begin{array}{l}1 \\
\text { fois/an }\end{array}$ & + & + & 2 à 3 ans & $\begin{array}{l}3 \text { à } 12 \text { mois } \\
\text { après le } \\
\text { début du } \\
\text { traitement }\end{array}$ & $\begin{array}{l}\text { Si perte de } \\
\text { taille/rachialgies }\end{array}$ \\
\hline Acide zolédronique & + & + & $\begin{array}{l}1 \\
\text { fois/an }\end{array}$ & + & + & 3 ans & $\begin{array}{l}\text { Si doute sur } \\
\text { la réalisation } \\
\text { de la } \\
\text { perfusion }\end{array}$ & $\begin{array}{l}\text { Si perte de } \\
\text { taille/rachialgies }\end{array}$ \\
\hline Denosumab & + & + & $\begin{array}{l}1 \\
\text { fois/an }\end{array}$ & + & + & 3 ans & $\begin{array}{l}\text { Si doute sur } \\
\text { la réalisation } \\
\text { de l'injection }\end{array}$ & $\begin{array}{l}\text { Si perte de } \\
\text { taille/rachialgies }\end{array}$ \\
\hline Teriparatide & + & + & $\begin{array}{l}1 \\
\text { fois/an }\end{array}$ & + & + & 18 mois & NON & $\begin{array}{l}\text { Si perte de } \\
\text { taille/rachialgies }\end{array}$ \\
\hline Raloxifène & + & + & $\begin{array}{l}1 \\
\text { fois/an }\end{array}$ & + & + & 2 à 3 ans & $\begin{array}{l}3 \text { à } 12 \text { mois } \\
\text { après le } \\
\text { début du } \\
\text { traitement }\end{array}$ & $\begin{array}{l}\text { Si perte de } \\
\text { taille/rachialgies }\end{array}$ \\
\hline THM & + & + & $\begin{array}{l}1 \\
\text { fois/an }\end{array}$ & + & + & 2 à 3 ans & $\begin{array}{l}3 \text { à } 12 \text { mois } \\
\text { après le } \\
\text { début du } \\
\text { traitement }\end{array}$ & $\begin{array}{l}\text { Si perte de } \\
\text { taille/rachialgies }\end{array}$ \\
\hline
\end{tabular}

+ : à réaliser à chaque consultation 
Figure 1 : seuil d'intervention (traitement pharmacologique) en fonction de la valeur du FRAX® pour fracture majeure de l'ostéoporose selon l'âge pour la France.

Risque de fracture

majeure à 10 ans (\%)

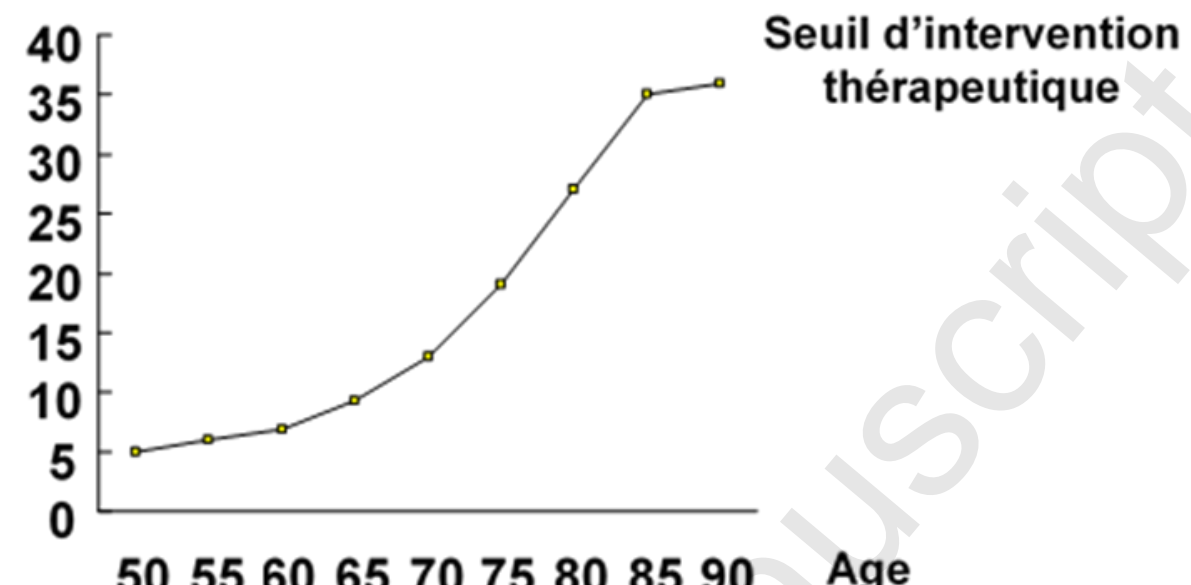

$\begin{array}{llllllllll}\text { Age } & \mathbf{5 0} & \mathbf{5 5} & \mathbf{6 0} & \mathbf{6 5} & \mathbf{7 0} & \mathbf{7 5} & \mathbf{8 0} & \mathbf{8 5} & \mathbf{9 0} \\ \% & 5 & 6 & 6,9 & \mathbf{9 , 3} & 13 & 19 & 27 & 35 & 36\end{array}$


Figure 2 : Indications thérapeutiques dans l'ostéoporose post-ménopausique

\begin{tabular}{|c|c|c|c|}
\hline $\begin{array}{c}\text { En fonction de la } \\
\text { diminution du T score (au } \\
\text { site le plus bas) }\end{array}$ & $\begin{array}{l}\text { Fractures sévères } \\
\text { (fémur, vertèbres } \\
\text { humérus, } \\
\text { bassin, tibia proximal) }\end{array}$ & Fractures non sévères & $\begin{array}{l}\text { Absence de fracture et } \\
\text { facteurs de risque } \\
\text { d'ostéoporose et/ou de } \\
\text { chutes multiples) }\end{array}$ \\
\hline $\mathrm{T}>-1$ & Avis du spécialiste & Pas de traitement & Pas de traitement \\
\hline$T \leq-1$ et $>-2$ & Traitement & Avis du spécialiste & Pas de traitement \\
\hline $\mathrm{T} \leq-2$ et $>-3$ & Traitement & Traitement & Avis du spécialiste \\
\hline$T \leq-3$ & Traitement & Traitement & Traitement \\
\hline
\end{tabular}

Table S1: Tableau des équivalences calciques (www.grio.org) 


\section{Tableau d'équivalences calciques}

Les valeurs sont données en milligrammes $(\mathrm{mg})$ de calcium pour une portion moyenne, dont le poids (avant cuisson) ou le volume est indiqué entre parenthèses.

Une grosse portion $=$ une portion moyenne $\times 1,5$

Une petite portion = une portion moyenne $\times 0,5$

\begin{tabular}{|c|c|c|c|c|}
\hline 1 & \multicolumn{3}{|c|}{ Lait : 1 verre $(100 \mathrm{ml})=1$ tasse $\quad(1 \mathrm{bol}=3$ verres $)$} & $120 \mathrm{mg}$ \\
\hline 2 & \multicolumn{3}{|c|}{1 yaourt $(125 \mathrm{~g})=1$ crème dessert } & $150 \mathrm{mg}$ \\
\hline 3 & \multicolumn{3}{|c|}{ Fromage blanc $(100 \mathrm{~g})=1$ pot individuel } & $95 \mathrm{mg}$ * \\
\hline \multirow[t]{2}{*}{4} & \multirow{2}{*}{$\begin{array}{l}\text { Petit } \\
\text { suisse }\end{array}$} & \multicolumn{2}{|l|}{ petit modèle } & $28 \mathrm{mg}$ \\
\hline & & \multicolumn{2}{|l|}{ grand modèle } & $56 \mathrm{mg}$ \\
\hline 5 & \multicolumn{3}{|c|}{ Fromage à pâte cuite $(30 \mathrm{~g})$} & $263 \mathrm{mg}$ * \\
\hline 6 & \multicolumn{3}{|c|}{ Fromage à pâte molle (30 g) } & $120 \mathrm{mg}$ * \\
\hline 7 & \multicolumn{3}{|c|}{ Viande et poisson $(120 \mathrm{~g})$} & $15 \mathrm{mg}$ * \\
\hline 8 & \multicolumn{3}{|l|}{1 oeuf } & $28 \mathrm{mg}$ \\
\hline 9 & \multicolumn{3}{|c|}{ Pommes de terre $(200 \mathrm{~g})$} & $20 \mathrm{mg}$ \\
\hline 10 & \multicolumn{3}{|c|}{ Frites $(160 \mathrm{~g})$} & $96 \mathrm{mg}$ \\
\hline 11 & \multicolumn{3}{|c|}{ Pâtes $(50 \mathrm{~g})=$ semoule } & $10 \mathrm{mg}$ \\
\hline 12 & \multicolumn{3}{|c|}{ Légumes secs $(75 \mathrm{~g})$} & $53 \mathrm{mg}$ * \\
\hline 13 & \multicolumn{3}{|c|}{ Légumes verts $(200 \mathrm{~g})$} & $94 \mathrm{mg}$ * \\
\hline \multirow[t]{3}{*}{14} & \multirow{3}{*}{ Pain } & \multicolumn{2}{|l|}{1 ficelle $(100 \mathrm{~g})$} & $25 \mathrm{mg}$ \\
\hline & & \multicolumn{2}{|l|}{1 baguette $(200 \mathrm{~g})$} & $50 \mathrm{mg}$ \\
\hline & & \multicolumn{2}{|l|}{1 biscotte $(10 \mathrm{~g})$} & $4 \mathrm{mg}$ \\
\hline 15 & \multicolumn{3}{|c|}{1 Fruit $(150 \mathrm{mg})$} & $25 \mathrm{mg}$ * \\
\hline \multirow[t]{4}{*}{$16 \& 17$} & \multirow{4}{*}{ Chocolat } & \multicolumn{2}{|c|}{1 barre de chocolat au lait ou blanc $(20 \mathrm{~g})$} & $43 \mathrm{mg}$ \\
\hline & & \multicolumn{2}{|c|}{1 barre de chocolat noir $(20 \mathrm{~g})$} & $13 \mathrm{mg}$ \\
\hline & & \multicolumn{2}{|c|}{1 tablette de chocolat au lait ou blanc $(100 \mathrm{~g})$} & $214 \mathrm{mg}$ \\
\hline & & \multicolumn{2}{|c|}{1 tablette de chocolat noir $(100 \mathrm{~g})$} & $63 \mathrm{mg}$ \\
\hline 18 & \multicolumn{3}{|c|}{ Eau du robinet $(100 \mathrm{ml})=1$ verre } & $9 \mathrm{mg}$ \\
\hline \multirow[t]{8}{*}{19} & & & Badoit & $22 \mathrm{mg}$ \\
\hline & & & Evian & $8 \mathrm{mg}$ \\
\hline & & & Contrexéville & $46 \mathrm{mg}$ \\
\hline & Fauminé & e nour un verre $(100 \mathrm{ml}$ & Perrier & $14 \mathrm{mg}$ \\
\hline & Lav & 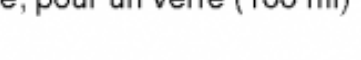 & Vichy Saint Yorre & $8 \mathrm{mg}$ \\
\hline & & & Hépar & $55 \mathrm{mg}$ \\
\hline & & & Vittel Grande Source & $20 \mathrm{mg}$ * \\
\hline & & & autre ou non précisée & $10 \mathrm{mg}$ * \\
\hline 20 & Autres bo & sons : 1 verre de vin, bière & $e$, jus de fruit & $7 \mathrm{mg}$ \\
\hline
\end{tabular}

* Représente la moyenne de plusieurs aliments du même groupe. 
